\title{
Rehabilitation interventions to support return to work for women with breast cancer: a systematic review and meta-analysis
}

\author{
Naomi Algeo ( $\square$ nalgeo@tcd.ie) \\ Discipline of Occupational Therapy, Trinity College Dublin \\ Kathleen Bennett \\ Data Science Centre, Royal College of Surgeons in Ireland, Dublin \\ Deirdre Connolly \\ Discipline of Occupational Therapy, Trinity College Dublin
}

\section{Research Article}

Keywords: employment, cancer survivorship, breast cancer, rehabilitation

Posted Date: March 3rd, 2021

DOI: https://doi.org/10.21203/rs.3.rs-250979/v1

License: (c) (i) This work is licensed under a Creative Commons Attribution 4.0 International License. Read Full License

Version of Record: A version of this preprint was published at BMC Cancer on August 5th, 2021. See the published version at https://doi.org/10.1186/s12885-021-08613-x. 


\section{Abstract \\ Background}

Research recommends the development and evaluation of interventions to support women with breast cancer in returning to, or managing, work. Despite this, there has historically been a paucity of rehabilitation interventions to support women with breast cancer to maintain or return to their work role.

The aim of this systematic review was to examine key characteristics of rehabilitation interventions, and their effectiveness on work outcomes for women with breast cancer, compared to usual care.

\section{Methods}

A systematic review was conducted of controlled studies of rehabilitation interventions with work outcomes for women with breast cancer. Six databases were systematically searched: EMBASE, Web of Science, MEDLINE (OVID), CINAHL, PsycINFO, and the Cochrane Central Register of Controlled Trials (CENTRAL). Results are presented either as pooled odds ratio (OR) or pooled effect size (hedges g) between groups, with $95 \%$ confidence intervals $(\mathrm{Cl})$. Narrative synthesis was conducted on intervention outcomes not suitable for meta-analysis.

\section{Results}

Five thousand, five hundred and thirty-five studies were identified. Nine out of 28 abstracts met inclusion criteria. Heterogeneity of interventions and outcomes precluded meta-analysis for most outcomes. Of the interventions included in meta-analysis, no significant differences compared to usual care were found for sick leave ( 2 studies ( 12 months); OR 1.11 ( $95 \%$ Cl: 0.66 to 1.87), number of sick days taken ( 2 studies (six months); difference in effect: -0.08 , ( $95 \% \mathrm{Cl}:-0.48$ to 0.38 ) or working hours ( 2 studies ( 12 months); 0.19 , ( $95 \% \mathrm{Cl}$ : -0.20 to 0.64 ). Only one study, with a multidisciplinary intervention, showed a significant difference for work outcomes when compared to usual care. Work-specific content featured in three interventions only, none of which provided conclusive evidence for improvement in work outcomes. Enhanced physical and psychological sequalae, and quality of life was observed in some studies.

\section{Conclusion}

There remains a lack of effective and methodologically rigorous rehabilitation intervention studies for breast cancer survivors. The development and evaluation of effective rehabilitation interventions to support return to work is warranted.

\section{Introduction}

Breast cancer accounted for over two million new cases in 2018 worldwide [1]. Survival is increasing, largely due to advancing treatments and earlier detection, and is as high as $85-90 \%$ at five-years in developed countries [2-4]. In line with increasing survivorship, there is a focus on optimising quality of life (QoL), for those living with and beyond cancer, including return to work (RTW). RTW rates vary across cancer types, and are influenced by personal, societal, workplace, healthcare, and legislative systems [5]. Typically, the one-year time point can be a milestone, where the mean delay in RTW has previously been reported at 11.4 months, however varying rates have been reported [6-7]. This could be related to disease and treatment-related factors which are often cited as RTW barriers, in addition to health-related QoL (HRQoL), cancer-related fatigue, cognitive dysfunction, and depression and anxiety [8-10].

A Cochrane review, providing evidence for vocational interventions to support RTW, reported moderate-quality evidence for multidisciplinary interventions to enhance work outcomes for all cancer cohorts including breast cancer, yet found it 'remarkable' that there remains a paucity in vocational interventions [11]. Vocational interventions have previously demonstrated promising outcomes for those living with chronic conditions such as heart disease, mental health disorders and intellectual disabilities [12-14]. Despite potential to enhance work outcomes for women with breast cancer, a previous systematic review yielded only four intervention studies, three of which were uncontrolled [15]. The aim of this study, therefore, was to systematically review rehabilitation intervention studies for women with breast cancer in relation to content, delivery and effectiveness of interventions on at least one work outcome when compared to usual care. Outcome measurements and theoretical frameworks underpinning interventions were also explored.

\section{Methods}


This review is reported as per Preferred Reporting Items for Systematic reviews and Meta-Analyses (PRISMA) checklist [16]. An initial review protocol was registered on the International Prospective Register of Systematic Reviews (PROSPERO) [ID: CRD42019145557] prior to commencing the review. However, as only one work-specific intervention study [17] was identified in the initial search, inclusion criteria were expanded to include rehabilitation interventions that measured the impact of the intervention on one or more work-related outcomes.

\section{Eligibility Criteria}

Inclusion criteria included (i) experimental designs including randomised control trials (RCTs) and quasi-experimental designs (with a comparator), (ii) group, individual and/or online interventions for women with breast cancer, (iii) a minimum of one work outcome reported, and (iv) in English.

\section{Information sources and search strategy}

A search strategy was developed with a medical librarian, and applied to EMBASE, Web of Science, MEDLINE (OVID), CINAHL, PsycINFO, and the Cochrane Central Register of Controlled Trials. Backwards and forwards chaining of all full-texts was also completed to identify reviews which were relevant but did not meet criteria for full-text review.

\section{Search selection}

Abstracts and titles of retrieved studies were screened by one reviewer. Where uncertainty remained, the study was examined in the full-text review to determine eligibility. Three reviewers were involved in full-text review. Where disagreement occurred between two reviewers regarding article inclusion/exclusion, a third reviewer intervened. Once full-text review was complete, data were extracted from included studies. EndNote was used to manage all retrieved studies, and Covidence for screening and data extraction.

\section{Data collection process and data items extracted}

A data extraction tool based on the Cochrane Handbook for Systematic Review of Interventions [18] was applied to independently extract data from each study onto an excel spreadsheet. Items recorded included

- Author(s), year of publication

- Study-design, setting, inclusion/exclusion criteria

- Type of intervention: format, duration, content, and facilitators

- Comparator

- Theoretical framework (as per Medical Research Council framework for complex interventions [19]).

- Outcomes: primary (work) and secondary (physical, psychological, and QoL) outcomes, outcome measures, and follow-up periods.

\section{Risk of bias in individual studies}

Two reviewers assessed risk of bias of each study using the Cochrane Handbook for Systematic Reviews of Interventions [18]. In cases of disagreement, the two reviewers discussed, with a third reviewer available for any unresolved disagreements.

\section{Summary measures}

Where outcomes were continuous, the estimated effect size was calculated from each published study using mean differences and standard deviations from each group (intervention and control) to calculate a standardised effect size using Hedges $g$ formula. For binary outcomes, odds ratios were used.

\section{Synthesis of results}

A meta-analysis of primary and secondary outcomes was planned if sufficient information was available and the studies were not too heterogeneous in relation to interventions, study designs, outcomes and measures of effect. If statistical synthesis was not possible, a narrative synthesis was planned to be conducted.

\section{Heterogeneity and pooling (meta-analysis) across studies}

$1^{2}$ index was used for the percentage of variance in meta-analysis attributable to study heterogeneity. However, this should be interpreted cautiously when a meta-analysis has few studies and can provide substantial bias, in which case confidence intervals (Cls) should 
supplement biased point estimate $\mathrm{I}^{2}$ [20]. The $\mathrm{H}^{2}$ statistic was also examined, where 1 is equal to perfect study homogeneity. The $\mathrm{H}^{2}$ statistic was considered where there were common measures across studies that could be pooled. In the case of binary outcomes, odds ratios (OR) and 95\% Cls were extracted or calculated for each study from available data. In the presence of significant heterogeneity, metaanalysis was performed using a random effects approach. Penalised likelihood is used for computing $95 \%$ confidence intervals for continuous measures. For pooling ORs the peto method was used for fixed (or random) effects.

\section{Results}

Using the search strategy, 5,535 records were identified, of which 28 papers met the inclusion criteria for full-text review (Fig. 1). Nine of the 28 studies were included in final synthesis. Of the 19 excluded papers, reasons for exclusion included (i) no work outcomes ( $n=15$ ), (ii) study-design other than RCTs or quasi-experimental designs (with comparator) $(n=3)$, and (iii) no clear reporting of work outcomes ( $n=1)$.

\section{Study Characteristics}

Of the nine included studies, all were RCT in design, three of which were pilot RCTs (Table 1$)$. Most studies ( $n=6)$ were published since 2010 with the remaining three studies spread across the 1980s, 1990s, and 2000s. Six studies were set in Europe, two in Canada, and one in the United States. Most interventions were delivered in a hospital setting $(n=6)$. One study did not specify context of intervention delivery however indicated that the intervention was partially home-based [21]. 
Table 1

Study and Participant Characteristics of included studies

\begin{tabular}{|c|c|c|c|c|c|c|c|}
\hline \multicolumn{5}{|c|}{ Study Characteristics } & \multicolumn{3}{|c|}{ Participant Characteristics } \\
\hline Author & Year & Design & Country & Setting & $\begin{array}{l}\mathrm{N} \text { (at } \\
\text { allocation) }\end{array}$ & Inclusion Criteria & Age \\
\hline $\begin{array}{l}\text { Björneklett } \\
\text { et al }\end{array}$ & 2013 & $\mathrm{RCT}$ & Sweden & $\begin{array}{l}\text { Resort } \\
\text { (type not } \\
\text { specified) }\end{array}$ & $\begin{array}{l}382 \\
\text { Intervention } \\
=191 \\
\text { Control = } \\
191\end{array}$ & $\begin{array}{l}\text { - Newly diagnosed primary breast } \\
\text { cancer } \\
\text { - No previous malignancy } \\
\text { - The physical and mental } \\
\text { capability to participate in group } \\
\text { interventions and to fill in } \\
\text { questionnaire } \\
\text { - Expected survival time of > } 12 \\
\text { months } \\
\text { - Analyses limited to those under } \\
\text { the age of } 65 \text { years old. }\end{array}$ & $\begin{array}{l}\text { Overall = } \\
\text { Unknown } \\
\text { Intervention } \\
=57.8 \\
\text { Control = } \\
58.7\end{array}$ \\
\hline $\begin{array}{l}\text { Bolam et } \\
\text { al }\end{array}$ & 2019 & RCT & Sweden & Hospital & $\begin{array}{l}240 \\
\text { RT-HIIT }{ }^{1}= \\
79 \\
\text { AT-HIIT }{ }^{2}= \\
80 \\
\text { Control = } 81\end{array}$ & $\begin{array}{l}\text { - Women } \\
\text { - 18-70 years } \\
\text { - Stage I-Illa breast cancer } \\
\text { - Scheduled to receive } \\
\text { chemotherapy directly }\end{array}$ & $\begin{array}{l}\text { Overall: } \\
\text { Unknown } \\
\text { RT-HIIT = } \\
\text { 52.7 AT- } \\
\text { HIIT = 54.4 } \\
\text { Control = } \\
52.6\end{array}$ \\
\hline $\begin{array}{l}\text { Hubbard } \\
\text { et al }\end{array}$ & 2013 & $\begin{array}{l}\text { Pilot } \\
\text { RCT }\end{array}$ & $\begin{array}{l}\text { Scotland, } \\
\text { UK }\end{array}$ & $\begin{array}{l}\text { Hospital } \\
\text { and } \\
\text { Community }\end{array}$ & $\begin{array}{l}22 \\
\text { Intervention } \\
=8 \\
\text { Control }=14\end{array}$ & $\begin{array}{l}\text { - 18-65 years } \\
\text { - In paid employment or self- } \\
\text { employed } \\
\text { - Living or working in Lothian or } \\
\text { Tayside, Scotland, UK } \\
\text { - Diagnosed with invasive breast } \\
\text { cancer tumour or ductal carcinoma } \\
\text { in situ } \\
\text { - Treated first with surgery }\end{array}$ & $\begin{array}{l}\text { Overall = } \\
50.5 \\
\text { Intervention } \\
=49.7 \\
\text { Control = } \\
51.0\end{array}$ \\
\hline $\begin{array}{l}\text { lbrahim et } \\
\text { al }\end{array}$ & 2017 & $\begin{array}{l}\text { Pilot } \\
\text { RCT }\end{array}$ & Canada & $\begin{array}{l}\text { Community } \\
\text { (Cancer } \\
\text { Support } \\
\text { Centre) }\end{array}$ & Control $=30$ & $\begin{array}{l}\text { - Scheduled to receive post- } \\
\text { operative adjuvant treatment } \\
\text { - Have an ECOG performance status } \\
0-1 \text {. }\end{array}$ & $\begin{array}{l}\text { Control = } \\
\text { Unknown }\end{array}$ \\
\hline Jong et al & 2018 & RCT & $\begin{array}{l}\text { The } \\
\text { Netherlands }\end{array}$ & $\begin{array}{l}\text { Hospital } \\
\text { and Home }\end{array}$ & $\begin{array}{l}83 \\
\text { Intervention } \\
=47 \\
\text { Control = } 36\end{array}$ & $\begin{array}{l}\text { - Women between 18-70 years } \\
\text { - Stage I-III breast cancer } \\
\text { - Scheduled for (neo)adjuvant } \\
\text { chemotherapy } \\
\text { - Able to understand and speak } \\
\text { Dutch } \\
\text { - Phone and internet access }\end{array}$ & $\begin{array}{l}\text { Overall: } \\
\text { Unknown } \\
\text { Intervention } \\
=51 \\
\text { Control = } 51\end{array}$ \\
\hline \multicolumn{8}{|c|}{$\begin{array}{l}{ }^{1} \mathrm{RT}-\mathrm{HIIT}=\text { Resistance Exercise and High-Intensity Interval Training; }{ }^{2} \text { AT-HIIT = Moderate Intensity Aerobic Exercise and High-Intensity } \\
\text { Interval Training }\end{array}$} \\
\hline Study Char & teristic & & & & Participant C & aracteristics & \\
\hline
\end{tabular}




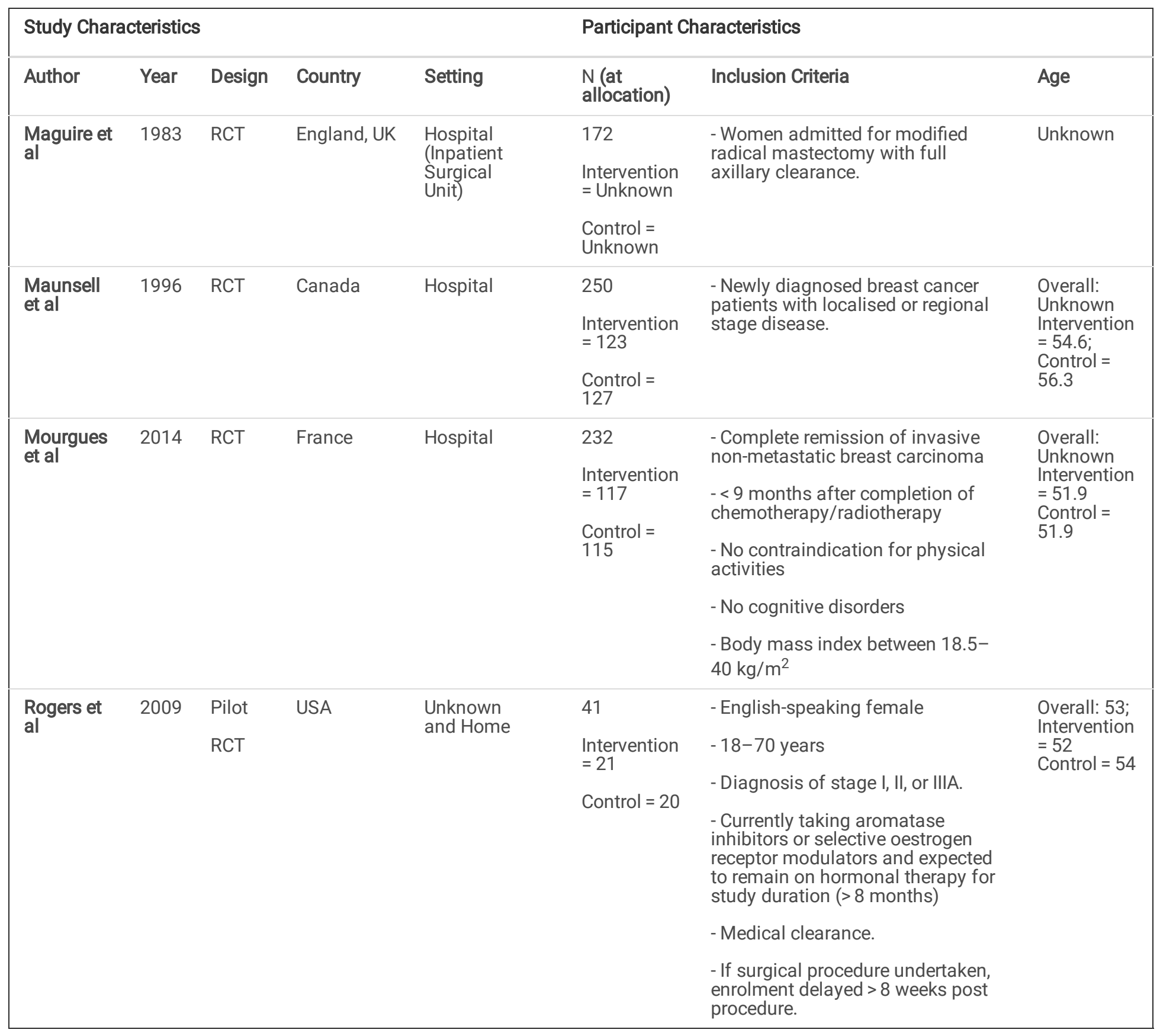

\section{Participants}

While all studies included women with breast cancer as an inclusion criterion, there were variations in eligibility criteria, including age (Table 1). Of the six studies specifying age as an inclusion criterion, three studies included 18-70 year olds. Three studies specified a staging criteria of stages I-III. Overall sample sizes ranged from 22 to 382 at allocation, [17, 22].

\section{Intervention characteristics:}

\section{Content}

Intervention content varied widely (Table 2). The majority of studies delivered a combination of physical and psychosocial interventions (n =6). Two studies delivered physical interventions and one study a psychosocial intervention. Only three interventions delivered workfocused components to the intervention including specific vocational guidance, encouragement to RTW and information on sick leave and insurance $[17,22,23]$. 
Table 2

Intervention Characteristics and Outcomes of included studies

\begin{tabular}{|c|c|c|c|c|c|c|}
\hline \multirow[t]{2}{*}{ Author } & \multicolumn{6}{|c|}{ Intervention Characteristics } \\
\hline & Format & Content & Facilitator(s) & Duration & $\begin{array}{l}\text { Theoretical } \\
\text { Framework }\end{array}$ & $\begin{array}{l}\text { Outcome } \\
\text { measures }\end{array}$ \\
\hline $\begin{array}{l}\text { Björneklett } \\
\text { et al } \\
(2013)\end{array}$ & $\begin{array}{l}\text { Face-to-face } \\
\text { Group. }\end{array}$ & $\begin{array}{l}\text { Physical/Psychosocial: } \\
\text { An information-based } \\
\text { programme } \\
\text { supplemented with } \\
\text { relaxation, qigong, } \\
\text { liberating dance, and } \\
\text { social activities. } \\
\text { Information sessions } \\
\text { included: } \\
\text { - Psychological reactions } \\
\text { to serious disease, \& } \\
\text { coping strategies. } \\
\text { - Practicalities of sick } \\
\text { leave from work, } \\
\text { insurance \& impact of } \\
\text { illness on finance } \\
\text { - Food and nutrition }\end{array}$ & $\begin{array}{l}\text { Oncologists, } \\
\text { social workers, a } \\
\text { psychologist, an } \\
\text { art therapist, } \\
\text { massage } \\
\text { therapists, a } \\
\text { dietician and a } \\
\text { person trained in } \\
\text { qigong and } \\
\text { mental } \\
\text { visualisation. }\end{array}$ & $\begin{array}{l}\text { One-week } \\
\text { inpatient stay } \\
\text { followed by } \\
\text { four-day } \\
\text { follow-up two } \\
\text { months later. } \\
\text { Duration of } \\
\text { individual } \\
\text { sessions not } \\
\text { specified. }\end{array}$ & None & $\begin{array}{l}\text { Sick Leave: } \\
\text { Single item } \\
\text { question } \\
\text { (Yes/No) and } \\
\text { number of days } \\
\text { taken for sick } \\
\text { leave. } \\
\text { Health care } \\
\text { utilisation: Asked } \\
\text { the frequency } \\
\text { and types of } \\
\text { healthcare visits. } \\
\text { Cost- } \\
\text { effectiveness } \\
\text { Measured at: } \\
\text { - } 2 \text { months } \\
\text { - } 6 \text { months } \\
\text { - } 12 \text { months }\end{array}$ \\
\hline $\begin{array}{l}\text { Bolam et } \\
\text { al (2019) }\end{array}$ & $\begin{array}{l}\text { Face-to-face } \\
\text { Group. }\end{array}$ & $\begin{array}{l}\text { Physical: } \\
\text { RT-HIIT }{ }^{1} \text { : Resistance } \\
\text { Exercises } \\
\text { using machine and free } \\
\text { weights followed by High } \\
\text { Intensity Interval Training } \\
\text { on a cycle ergometer. } \\
\text { AT-HIIT } 2 \text { : } 20 \text { min of } \\
\text { moderate intensity } \\
\text { continuous Aerobic } \\
\text { Exercise followed by HIIT } \\
\text { on a cycle ergometer. }\end{array}$ & $\begin{array}{l}\text { Exercise } \\
\text { physiologist, } \\
\text { oncology nurse. }\end{array}$ & $\begin{array}{l}60 \text {-minute } \\
\text { sessions } \\
\text { twice per } \\
\text { week on non- } \\
\text { consecutive } \\
\text { weekdays, } \\
\text { over } 16 \\
\text { weeks. }\end{array}$ & None & $\begin{array}{l}\text { Sick leave: Single } \\
\text { item question (\% } \\
\text { of leave taken; 0, } \\
25,50,75,100 \%) \\
\text { Cancer-related } \\
\text { fatigue: Revised } \\
\text { Piper Fatigue } \\
\text { Scale (PFS) } \\
\text { Quality of Life: } \\
\text { EORTC-QLQ-C301 } \\
\text { Symptom and } \\
\text { Symptom } \\
\text { Burden: } \\
\text { Memorial } \\
\text { Symptom } \\
\text { Assessment } \\
\text { Scale (MSAS) } \\
\text { Measured at: } \\
\text { - } 1 \text { Year } \\
\text { - } 2 \text { Years }\end{array}$ \\
\hline
\end{tabular}




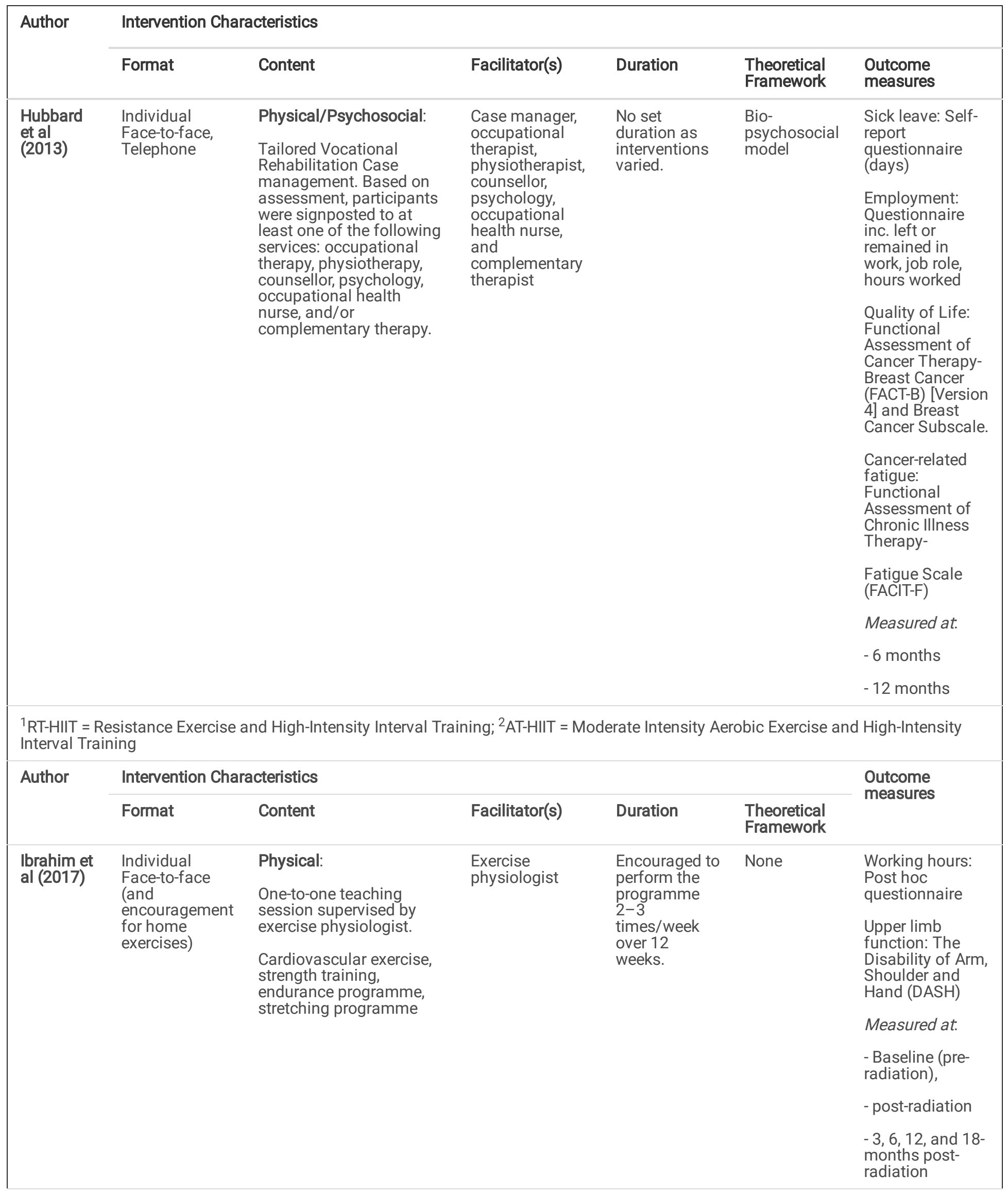




\begin{tabular}{|c|c|c|c|c|c|c|}
\hline \multirow[t]{2}{*}{ Author } & \multicolumn{6}{|c|}{ Intervention Characteristics } \\
\hline & Format & Content & Facilitator(s) & Duration & $\begin{array}{l}\text { Theoretical } \\
\text { Framework }\end{array}$ & $\begin{array}{l}\text { Outcome } \\
\text { measures }\end{array}$ \\
\hline \multirow{14}{*}{$\begin{array}{l}\text { Jong et al } \\
\text { (2018) }\end{array}$} & \multirow{14}{*}{$\begin{array}{l}\text { Face-to-Face } \\
\text { and Home- } \\
\text { Based work | } \\
\text { Group }\end{array}$} & Physical/Psychosocial & \multirow[t]{14}{*}{ Yoga instructors } & \multirow{14}{*}{$\begin{array}{l}\text { 75-minute } \\
\text { sessions once } \\
\text { a week for } 12 \\
\text { weeks. }\end{array}$} & \multirow[t]{14}{*}{ None } & \multirow{3}{*}{$\begin{array}{l}\text { Reintegration to } \\
\text { work: Assessed } \\
\text { via telephone } \\
\text { interview. } \\
\text { Returned to work } \\
\text { Binary Yes/No. } \\
\text { Fatigue: } \\
\text { Multidimensiona } \\
\text { Fatigue Inventory } \\
\text { [MFIl; Fatigue } \\
\text { Quality List [FQL] }\end{array}$} \\
\hline & & $\begin{array}{l}\text { A Dru-based Yoga. } \\
\text { Programme which } \\
\text { includes } 15 \text {-minute blocks } \\
\text { of the following: }\end{array}$ & & & & \\
\hline & & & & & & \\
\hline & & - Breathing awareness & & & & \multirow{2}{*}{$\begin{array}{l}\text { Quality of Life: } \\
\text { EORTC-QLQ-C- } \\
30^{1}\end{array}$} \\
\hline & & - Energy block release & & & & \\
\hline & & - Body awareness & & & & \multirow{3}{*}{$\begin{array}{l}\text { Psychological } \\
\text { Distress: } \\
\text { Hospital Anxiety } \\
\text { Depression Scale } \\
\text { [HADS]; }\end{array}$} \\
\hline & & - Relaxation & & & & \\
\hline & & \multirow{7}{*}{$\begin{array}{l}\text { In addition, women were } \\
\text { provided a CD/MP3 } \\
\text { download with 20-minute } \\
\text { relaxation and breathing } \\
\text { exercises to complete at } \\
\text { home. }\end{array}$} & & & & \\
\hline & & & & & & $\begin{array}{l}\text { Impact of Events } \\
\text { Scale [IES] }\end{array}$ \\
\hline & & & & & & $\begin{array}{l}\text { Treatment } \\
\text { expectations: } \\
\text { Participants } \\
\text { Expectations } \\
\text { questionnaire. }\end{array}$ \\
\hline & & & & & & Measured at. \\
\hline & & & & & & - Baseline (T0) \\
\hline & & & & & & - 3 months (T1) \\
\hline & & & & & & - 6 months (T2) \\
\hline
\end{tabular}




\begin{tabular}{|c|c|c|c|c|c|c|}
\hline \multirow[t]{2}{*}{ Author } & \multicolumn{6}{|c|}{ Intervention Characteristics } \\
\hline & Format & Content & Facilitator(s) & Duration & $\begin{array}{l}\text { Theoretical } \\
\text { Framework }\end{array}$ & $\begin{array}{l}\text { Outcome } \\
\text { measures }\end{array}$ \\
\hline $\begin{array}{l}\text { Maguire et } \\
\text { al (1983) }\end{array}$ & $\begin{array}{l}\text { Individual } \\
\text { Face-to-face }\end{array}$ & $\begin{array}{l}\text { Physical/Psychosocial } \\
\text { Counselling/Education: } \\
\text { - Nurse advised range of } \\
\text { movement exercises for } \\
\text { arm. } \\
\text { - Encouragement to look } \\
\text { at and discuss scar and } \\
\text { loss of breast. } \\
\text { - Demonstration of } \\
\text { possible external breast } \\
\text { protheses. } \\
\text { - Home-visit post- } \\
\text { discharge to assess upper } \\
\text { limb monitor adherence } \\
\text { to exercises and } \\
\text { counselling. } \\
\text { - Encouragement of return } \\
\text { to work and social } \\
\text { reintegration. }\end{array}$ & Nurse specialist & $\begin{array}{l}\text { Throughout } \\
\text { inpatient stay } \\
\text { post-surgery } \\
\text { (varied among } \\
\text { participants). } \\
\text { Followed up } \\
\text { at home visit } \\
\text { every two } \\
\text { months until } \\
\text { deemed fit for } \\
\text { discharge. }\end{array}$ & None & $\begin{array}{l}\text { RTW: Yes/No/ } \\
\text { Non-Applicable } \\
\text { Response to scar, } \\
\text { prosthesis and } \\
\text { breast loss: } \\
\text { Interview } \\
\text { response } \\
\text { (satisfied, } \\
\text { neutral, } \\
\text { dissatisfied) } \\
\text { Perceived Impact } \\
\text { on Swelling, } \\
\text { Pain, and } \\
\text { Disability: Self } \\
\text { report } \\
\text { Social } \\
\text { adjustment: } \\
\text { Single item } \\
\text { question on } \\
\text { problems with } \\
\text { social } \\
\text { adjustment } \\
\text { Housework: } \\
\text { Single item } \\
\text { question on } \\
\text { problems with } \\
\text { housework } \\
\text { Marital } \\
\text { adjustment: } \\
\text { Concurrent } \\
\text { physical illness: } \\
\text { Measured at. } \\
\text { - } 3 \text { months } \\
\text { - } 12 \text { months } \\
\text { - } 18 \text { months }\end{array}$ \\
\hline \multirow[t]{2}{*}{ Author } & \multicolumn{6}{|c|}{ Intervention Characteristics } \\
\hline & Format & Content & Facilitator(s) & Duration & $\begin{array}{l}\text { Theoretical } \\
\text { Framework }\end{array}$ & $\begin{array}{l}\text { Outcome } \\
\text { measures }\end{array}$ \\
\hline
\end{tabular}




\begin{tabular}{|c|c|c|c|c|c|c|}
\hline \multirow[t]{2}{*}{ Author } & \multicolumn{6}{|c|}{ Intervention Characteristics } \\
\hline & Format & Content & Facilitator(s) & Duration & $\begin{array}{l}\text { Theoretical } \\
\text { Framework }\end{array}$ & $\begin{array}{l}\text { Outcome } \\
\text { measures }\end{array}$ \\
\hline \multirow{12}{*}{$\begin{array}{l}\text { Maunsell } \\
\text { et al } \\
(1996)\end{array}$} & Individual & Psychosocial: & \multirow[t]{12}{*}{ Social worker } & \multirow{12}{*}{$\begin{array}{l}\text { Telephone } \\
\text { screening } \\
\text { every } 28 \text { days } \\
\text { for total of } 12 \\
\text { screening } \\
\text { calls. }\end{array}$} & \multirow{12}{*}{$\begin{array}{l}\text { Brief crisis } \\
\text { intervention } \\
\text { model. }\end{array}$} & RTW: Binary \\
\hline & \multirow{11}{*}{$\begin{array}{l}\text { Face-to-face } \\
\text { and Telephone }\end{array}$} & \multirow{11}{*}{$\begin{array}{l}\text { Interventions included } \\
\text { mix of information, } \\
\text { education, support, } \\
\text { counselling and referral } \\
\text { where required. }\end{array}$} & & & & to work \\
\hline & & & & & & $\begin{array}{l}\text { Working } \\
\text { hours/week: } \\
\text { Number of hours. }\end{array}$ \\
\hline & & & & & & $\begin{array}{l}\text { Psychologic } \\
\text { symptoms: } \\
\text { General Health } \\
\text { Questionnaire } \\
\text { [GHQ] }\end{array}$ \\
\hline & & & & & & $\begin{array}{l}\text { Psychologic } \\
\text { distress: } \\
\text { Psychiatric } \\
\text { Symptom Index }\end{array}$ \\
\hline & & & & & & $\begin{array}{l}\text { Social support: } \\
\text { Social Support } \\
\text { Questionnaire }\end{array}$ \\
\hline & & & & & & $\begin{array}{l}\text { Stressful Life } \\
\text { Events: Life } \\
\text { Experiences } \\
\text { Survey }\end{array}$ \\
\hline & & & & & & $\begin{array}{l}\text { Marital } \\
\text { satisfaction: The } \\
\text { Locke-Wallace } \\
\text { Marital } \\
\text { Adjustment Test } \\
\text { [LWMAT] }\end{array}$ \\
\hline & & & & & & $\begin{array}{l}\text { Depression and } \\
\text { Anxiety: } \\
\text { Diagnostic } \\
\text { Interview } \\
\text { Schedule [DIS] }\end{array}$ \\
\hline & & & & & & $\begin{array}{l}\text { Physical Health: } \\
\text { Self-report }\end{array}$ \\
\hline & & & & & & $\begin{array}{l}\text { Outcomes } \\
\text { measured: }\end{array}$ \\
\hline & & & & & & $\begin{array}{l}\text { Baseline (T0), } 3 \\
\text { months (T1), } 6 \\
\text { months (T2) }\end{array}$ \\
\hline \multirow{4}{*}{$\begin{array}{l}\text { Mourgues } \\
\text { et al } \\
\text { (2014) }\end{array}$} & \multirow{4}{*}{$\begin{array}{l}\text { Face-to-face } \\
\text { Group }\end{array}$} & \multirow{4}{*}{$\begin{array}{l}\text { Physical/Psychosocial } \\
\text { Multicomponent } \\
\text { including physiotherapy, } \\
\text { nutritional advice, thermal } \\
\text { water treatment, daily } \\
\text { two-hour physical activity, } \\
\text { running and basic dietary } \\
\text { follow-up. Consultation } \\
\text { with dietitian every six } \\
\text { months. }\end{array}$} & \multirow{4}{*}{$\begin{array}{l}\text { Physiotherapist, } \\
\text { Dietitian, }\end{array}$} & \multirow{4}{*}{$\begin{array}{l}\text { 15-day } \\
\text { programme. } \\
\text { Daily two-hour } \\
\text { physical } \\
\text { activity. }\end{array}$} & \multirow[t]{4}{*}{ None } & \multirow{2}{*}{$\begin{array}{l}\text { Occupational } \\
\text { activity: Total } \\
\text { hourly volume of } \\
\text { overall \& } \\
\text { occupational } \\
\text { activity. }\end{array}$} \\
\hline & & & & & & \\
\hline & & & & & & $\begin{array}{l}\text { Daily abilities: } \\
\text { Perception } \\
\text { whether health } \\
\text { problems } \\
\text { impacted on } \\
\text { activities. }\end{array}$ \\
\hline & & & & & & $\begin{array}{l}\text { Outcomes } \\
\text { measured: } \\
\text { Baseline, } 6 \text { \& } \\
\text { 12months }\end{array}$ \\
\hline
\end{tabular}




\begin{tabular}{|c|c|c|c|c|c|c|}
\hline \multirow[t]{2}{*}{ Author } & \multicolumn{6}{|c|}{ Intervention Characteristics } \\
\hline & Format & Content & Facilitator(s) & Duration & $\begin{array}{l}\text { Theoretical } \\
\text { Framework }\end{array}$ & $\begin{array}{l}\text { Outcome } \\
\text { measures }\end{array}$ \\
\hline \multirow{12}{*}{$\begin{array}{l}\text { Rogers et } \\
\text { al (2009) }\end{array}$} & \multirow{12}{*}{$\begin{array}{l}\text { Face-to-Face } \\
\text { and home- } \\
\text { based } \\
\text { exercises | } \\
\text { Group and } \\
\text { Individual }\end{array}$} & Physical/Psychosocial & \multirow{12}{*}{$\begin{array}{l}\text { Clinical } \\
\text { Psychologist, } \\
\text { Exercise } \\
\text { specialists } \\
\text { (certified by } \\
\text { American } \\
\text { College of } \\
\text { Sports Medicine } \\
\text { or certified } \\
\text { eligible). }\end{array}$} & \multirow{12}{*}{$\begin{array}{l}\text { 12-week } \\
\text { programme. }\end{array}$} & \multirow{12}{*}{$\begin{array}{l}\text { Social } \\
\text { Cognitive } \\
\text { Theory }\end{array}$} & \multirow{2}{*}{$\begin{array}{l}\text { Sick days: Self- } \\
\text { report number of } \\
\text { days off work }\end{array}$} \\
\hline & & $\begin{array}{l}\text { The BEAT Cancer } \\
\text { programme: }\end{array}$ & & & & \\
\hline & & $\begin{array}{l}\text { - } 12 \text { individual supervised } \\
\text { exercise }\end{array}$ & & & & \multirow{3}{*}{$\begin{array}{l}\text { Quality of life: } \\
\text { Functional } \\
\text { Assessment of } \\
\text { Cancer Therapy- } \\
\text { Breast (FACT-B) } \\
\text { \& FACT-G } \\
\text { (General) }\end{array}$} \\
\hline & & - Home-based exercise & & & & \\
\hline & & - 3 individual face-to-face & & & & \\
\hline & & \multirow{7}{*}{$\begin{array}{l}\text { - Six discussion group } \\
\text { sessions addressing: } \\
\text { Social support, } \\
\text { Journaling, Time } \\
\text { Management, Stress } \\
\text { Management, Dealing } \\
\text { with Exercise Barriers, } \\
\text { Behaviour modification }\end{array}$} & & & & $\begin{array}{l}\text { Fatigue: FACT- } \\
\text { Fatigue (FACT-F) }\end{array}$ \\
\hline & & & & & & $\begin{array}{l}\text { Endocrine } \\
\text { symptoms: FACT } \\
\text {-Endocrine } \\
\text { Symptoms } \\
\text { (FACT-ES) }\end{array}$ \\
\hline & & & & & & $\begin{array}{l}\text { Cognitive } \\
\text { function: FACT- } \\
\text { Cognitive }\end{array}$ \\
\hline & & & & & & $\begin{array}{l}\text { Sleep } \\
\text { dysfunction: } \\
\text { Pittsburgh Sleep } \\
\text { Quality Index } \\
\text { (PSQI) }\end{array}$ \\
\hline & & & & & & $\begin{array}{l}\text { Physical activity } \\
\text { behaviour: The } \\
\text { Godin Leisure- } \\
\text { Time Exercise } \\
\text { Questionnaire }\end{array}$ \\
\hline & & & & & & $\begin{array}{l}\text { Motivational } \\
\text { readiness for } \\
\text { physical activity: } \\
\text { Self-report of } \\
\text { stage of change }\end{array}$ \\
\hline & & & & & & $\begin{array}{l}\text { Lower extremity } \\
\text { pain and } \\
\text { function: } \\
\text { Western Ontario } \\
\text { and McMaster } \\
\text { Universities } \\
\text { Arthritis Index } \\
\text { (WOMAC) }\end{array}$ \\
\hline
\end{tabular}

Format and delivery. All studies included face-to-face intervention delivery. Two studies also delivered the intervention partially by telephone. Three interventions including exercise involved home-based self-directed exercises. Four interventions were group-based, four were individual, and one intervention was blended (group and 1:1). Individual session length was not described in all papers, however, was usually indicated in physical interventions where session lengths varied between 60, 75, and 120 minutes (Table 2).

\section{Theoretical framework}

Most studies $(n=6)$ did not report a specific theoretical framework/model used to guide intervention design or delivery (Table 2). Only three studies reported application of theoretical frameworks. These included the Biopsychosocial Model [17], the Brief Crisis Intervention Model [24], and Social Cognitive Theory [21].

\section{Comparator}

All studies reported comparators of usual care (Table 2). This most frequently included provision of written materials (e.g. physical activity [21, 25]; 'Work and Cancer' [17]). Usual care also included encouragement of healthy lifestyles [26], nurse support [27], a psychologic 
follow-up programme and physiotherapy [24], or dietitian consultation [28].

\section{Outcomes and Outcome Measures}

All work outcomes were assessed by self-report (Table 2). The most commonly assessed work outcome was sick leave/RTW (binary yes/no question if the participant had returned to work in some capacity). The second most commonly measured outcome was number of working hours, followed by number of sick days. One study assessed occupational (work) capacity by asking women if their health problems adversely impacted on ability to complete occupational activities [28]. The most frequently measured patient-reported outcomes included physical $(n=7)$ and psychological $(n=6)$ sequalae, and QoL $(n=4)$. Other outcomes included sleep, symptom burden, household tasks, social activities, and marital adjustment. Outcome measures varied across studies, with little overlap in most cases.

\section{Risk of bias within studies}

A risk of bias assessment summary for each included study is indicated in Fig. 2. Every study was deemed high risk for blinding of participants and personnel as due to the nature of the intervention it was not possible to blind participants. Participants assessed their own outcomes (as using self-reported questionnaires) and so it is unclear if awareness of their randomised allocation might have directly influenced the outcomes. For further details, see Supplementary Material 1. 
Table 3

Results of individual studies included in the systematic review and meta-analysis

\begin{tabular}{|c|c|c|c|c|c|c|c|}
\hline \multirow[b]{2}{*}{ Author } & \multicolumn{3}{|c|}{ Work outcomes } & \multicolumn{4}{|l|}{ Other outcomes } \\
\hline & $\begin{array}{l}\text { Sick Leave } \\
\text { / RTW } \\
(\mathrm{Y} / \mathrm{N})\end{array}$ & $\begin{array}{l}\text { Working } \\
\text { hours }\end{array}$ & Other: & Physical & Psychological & QoL & Other \\
\hline $\begin{array}{l}\text { Björneklett } \\
\text { et al } \\
\text { (2013) }\end{array}$ & $\begin{array}{l}\text { (Sick } \\
\text { Leave - } \\
\text { Days): } \\
\text { No } \\
\text { significant } \\
\text { differences } \\
\text { at } 0,2,6 \text {, } \\
\text { or } 12 \\
\text { months. }\end{array}$ & & & & & & $\begin{array}{l}\text { Healthcare } \\
\text { utilisation: Not } \\
\text { significant re. } \\
\text { visits to medical } \\
\text { specialists, GPs } \\
\text { or } \\
\text { physiotherapists. } \\
\text { However, women } \\
\text { treated with } \\
\text { chemotherapy in } \\
\text { intervention } \\
\text { group had } \\
\text { significantly } \\
\text { more visits with } \\
\text { 'Other' } \\
\text { healthcare } \\
\text { professionals } \\
\text { than the control } \\
\text { at } 6 \text { and } 12 \\
\text { months. } \\
\text { Health } \\
\text { economics: } \\
\text { Intervention was } \\
\text { significantly } \\
\text { greater in cost } \\
\text { compared to } \\
\text { control. }\end{array}$ \\
\hline $\begin{array}{l}\text { Bolam et } \\
\text { al (2019) }\end{array}$ & $\begin{array}{l}\% \text { of sick } \\
\text { leave at } \\
\text { that } \\
\text { timepoint. } \\
\text { No } \\
\text { significant } \\
\text { differences } \\
\text { between } \\
\text { the two } \\
\text { groups at } \\
2 \text { years. }\end{array}$ & & & $\begin{array}{l}\text { Cancer-related } \\
\text { fatigue (CRF): } \\
\text { Significant } \\
\text { differences } \\
\text { between RT- } \\
\text { HIIT and } \\
\text { control } \\
\text { groups for } \\
\text { CRF and } \\
\text { Cognitive CRF } \\
\text { in favour of } \\
\text { RT-HIIT who } \\
\text { experienced } \\
\text { improvements } \\
\text { in both. } \\
\text { Physical } \\
\text { symptoms } \\
\text { (Item MSAS): } \\
\text { No significant } \\
\text { differences }\end{array}$ & $\begin{array}{l}\text { Psychological } \\
\text { symptoms } \\
\text { (Item of } \\
\text { MSAS): No } \\
\text { significant } \\
\text { differences } \\
\text { between } \\
\text { groups. } \\
\text { Emotional } \\
\text { functioning } \\
\text { (item of } \\
\text { EORTC): No } \\
\text { significant } \\
\text { differences }\end{array}$ & $\begin{array}{l}\text { QoL: No } \\
\text { significant } \\
\text { differences } \\
\text { between the } \\
\text { two groups. }\end{array}$ & $\begin{array}{l}\text { Total symptoms: } \\
\text { Sig. } \downarrow \text { total } \\
\text { symptoms than } \\
\text { UC at } 2 \text { years in } \\
\text { favour of AT-HIIT } \\
\text { intervention. } \\
\text { Symptom } \\
\text { burden: Sig. } \downarrow \\
\text { symptom burden } \\
\text { than UC at } 2 \\
\text { years in favour } \\
\text { of AT-HIIT } \\
\text { intervention. }\end{array}$ \\
\hline $\begin{array}{l}\text { Hubbard } \\
\text { et al } \\
(2013)\end{array}$ & $\begin{array}{l}\text { Number of } \\
\text { days sick } \\
\text { leave: } \\
\text { No } \\
\text { significant } \\
\text { differences }\end{array}$ & & $\begin{array}{l}\text { Change in } \\
\text { employment } \\
\text { pattern: No } \\
\text { significant } \\
\text { differences } \\
\text { between } \\
\text { groups }\end{array}$ & $\begin{array}{l}\text { Fatigue: No } \\
\text { significant } \\
\text { differences } \\
\text { Physical } \\
\text { functioning } \\
\text { (item of FACT- } \\
B \text { ): No } \\
\text { significant } \\
\text { differences }\end{array}$ & $\begin{array}{l}\text { Emotional } \\
\text { functioning } \\
\text { (item of FACT- } \\
B \text { ): No } \\
\text { significant } \\
\text { differences }\end{array}$ & $\begin{array}{l}\text { QoL: } \\
\text { Significant } \\
\text { differences } \\
\text { between } \\
\text { groups on } \\
\text { breast cancer } \\
\text { specific QoL in } \\
\text { favour of } \\
\text { intervention } \\
\text { who } \\
\text { experienced } \\
\text { improvements. }\end{array}$ & \\
\hline \multicolumn{8}{|c|}{$\begin{array}{l}\text { QoL: Quality of Life; RT-HIIT = Resistance Exercise and High-Intensity Interval Training; AT-HIIT = Moderate Intensity Aerobic Exercise } \\
\text { and High-Intensity Interval Training }\end{array}$} \\
\hline
\end{tabular}




\begin{tabular}{|c|c|c|c|c|c|c|c|}
\hline & \multicolumn{3}{|c|}{ Work outcomes } & \multicolumn{4}{|l|}{ Other outcomes } \\
\hline & \multicolumn{3}{|c|}{ Work outcomes } & \multicolumn{4}{|c|}{ Other outcomes } \\
\hline Author & $\begin{array}{l}\text { Sick Leave } \\
\text { / RTW } \\
(\mathrm{Y} / \mathrm{N})\end{array}$ & $\begin{array}{l}\text { Working } \\
\text { hours }\end{array}$ & Other: & Physical & Psychological & QoL & Other: \\
\hline $\begin{array}{l}\text { Ibrahim et } \\
\text { al (2017) }\end{array}$ & & $\begin{array}{l}\text { Not } \\
\text { reported } \\
\text { for control } \\
\text { group } \\
\text { therefore } \\
\text { unable to } \\
\text { ascertain if } \\
\text { significant. }\end{array}$ & & $\begin{array}{l}\text { Upper limb } \\
\text { function: No } \\
\text { significant } \\
\text { differences }\end{array}$ & & & \\
\hline $\begin{array}{l}\text { Jong et al } \\
\text { (2018) }\end{array}$ & $\begin{array}{l}\text { Return to } \\
\text { work } \\
(Y / N) \text { : No } \\
\text { significant } \\
\text { difference } \\
\text { between } \\
\text { groups }\end{array}$ & & & $\begin{array}{l}\text { Fatigue: No } \\
\text { significant } \\
\text { differences } \\
\text { Confidence in } \\
\text { fatigue } \\
\text { reduction: } \\
\text { Significantly } \\
\text { more } \\
\text { confident in } \\
\text { fatigue } \\
\text { reduction in } \\
\text { favour of } \\
\text { intervention } \\
\text { group. } \\
\text { Adequate } \\
\text { relief of } \\
\text { fatigue }(Y / N) \text { : } \\
\text { Significantly } \\
\text { more relief of } \\
\text { fatigue in } \\
\text { favour of } \\
\text { intervention } \\
\text { group. }\end{array}$ & $\begin{array}{l}\text { Psychological } \\
\text { distress: } \\
\text { No significant } \\
\text { differences in } \\
\text { levels of } \\
\text { anxiety } \\
\text { Significantly } \\
\text { less } \\
\text { depressive } \\
\text { symptoms at } \\
3 \text { months in } \\
\text { favour of } \\
\text { intervention. } \\
\text { Emotional } \\
\text { functioning } \\
\text { (item of } \\
\text { EORTC): No } \\
\text { significant } \\
\text { differences }\end{array}$ & $\begin{array}{l}\text { QoL: } \\
\text { Significantly } \\
\text { less nausea } \\
\text { and vomiting } \\
\text { at six months } \\
\text { in favour of } \\
\text { Intervention } \\
\text { group. No } \\
\text { significant } \\
\text { differences for } \\
\text { other } \\
\text { outcomes. }\end{array}$ & $\begin{array}{l}\text { Impact of } \\
\text { events: No } \\
\text { significant } \\
\text { differences. } \\
\text { Treatment } \\
\text { expectations: } \\
\text { Intervention } \\
\text { group had } \\
\text { significantly } \\
\text { higher treatment } \\
\text { expectations } \\
\text { compared to } \\
\text { control. }\end{array}$ \\
\hline $\begin{array}{l}\text { Maguire et } \\
\text { al (1983) }\end{array}$ & \multicolumn{2}{|l|}{$\begin{array}{l}R T W \\
(Y / N) \text { : No } \\
\text { significant } \\
\text { differences }\end{array}$} & & $\begin{array}{l}\text { Upper limb } \\
\text { swelling, pain } \\
\text { and disability } \\
\text { No significant } \\
\text { differences }\end{array}$ & $\begin{array}{l}\text { Reaction to } \\
\text { scar, } \\
\text { prosthesis } \\
\text { and breast } \\
\text { loss: } \\
\text { Intervention } \\
\text { group were } \\
\text { significantly } \\
\text { more } \\
\text { satisfied with } \\
\text { scar, } \\
\text { prothesis, } \\
\text { breast loss, } \\
\text { compared to } \\
\text { control. }\end{array}$ & & $\begin{array}{l}\text { Housework, } \\
\text { Social } \\
\text { adjustment, } \\
\text { Martial } \\
\text { adjustment: No } \\
\text { significant } \\
\text { differences } \\
\text { between groups. }\end{array}$ \\
\hline \multicolumn{8}{|c|}{$\begin{array}{l}\text { QoL: Quality of Life; RT-HIIT = Resistance Exercise and High-Intensity Interval Training; AT-HIIT = Moderate Intensity Aerobic Exercise } \\
\text { and High-Intensity Interval Training }\end{array}$} \\
\hline \multicolumn{4}{|c|}{ Work outcomes } & \multicolumn{4}{|l|}{ Other outcomes } \\
\hline Author & $\begin{array}{l}\text { Sick Leave } \\
\text { / RTW } \\
(\mathrm{Y} / \mathrm{N})\end{array}$ & $\begin{array}{l}\text { Working } \\
\text { hours }\end{array}$ & Other: & Physical & Psychological & QoL & Other: \\
\hline
\end{tabular}




\begin{tabular}{|c|c|c|c|c|c|c|c|}
\hline \multirow[b]{2}{*}{$\begin{array}{l}\text { Maunsell } \\
\text { et al } \\
\text { (1996) }\end{array}$} & \multicolumn{3}{|c|}{ Work outcomes } & \multicolumn{4}{|l|}{ Other outcomes } \\
\hline & $\begin{array}{l}R T W \\
(Y / N) \text { : } \\
\text { No } \\
\text { significant } \\
\text { differences }\end{array}$ & $\begin{array}{l}\text { Working } \\
\text { hours (per } \\
\text { week): No } \\
\text { significant } \\
\text { differences }\end{array}$ & & $\begin{array}{l}\text { Physical } \\
\text { health: No } \\
\text { significant } \\
\text { differences }\end{array}$ & $\begin{array}{l}\text { Psychological } \\
\text { distress: No } \\
\text { significant } \\
\text { differences }\end{array}$ & & $\begin{array}{l}\text { Perception of } \\
\text { health. } \\
\text { Functional } \\
\text { status, Social } \\
\text { activity, Marital } \\
\text { relations: No } \\
\text { significant } \\
\text { differences }\end{array}$ \\
\hline $\begin{array}{l}\text { Mourgues } \\
\text { et al } \\
\text { (2014) }\end{array}$ & & & $\begin{array}{l}\text { Occupational } \\
\text { activity } \\
\text { (Work): } \\
\text { Significant } \\
\text { improvement } \\
\text { in ability to } \\
\text { perform work } \\
\text { activities at } \\
12 \text { months in } \\
\text { favour of } \\
\text { intervention } \\
\text { group. }\end{array}$ & & & & $\begin{array}{l}\text { Overall activities: } \\
\text { Significant } \\
\text { differences } \\
\text { between groups } \\
\text { in favour of } \\
\text { intervention who } \\
\text { had increased } \\
\text { resumption of } \\
\text { overall activities } \\
\text { in first 12-month } \\
\text { period. } \\
\text { Non- } \\
\text { occupational } \\
\text { activity (Family, } \\
\text { household tasks } \\
\text { and } \\
\text { volunteerism): } \\
\text { Significant } \\
\text { improvement in } \\
\text { ability to perform } \\
\text { family activities } \\
\text { at } 12 \text { months, in } \\
\text { favour of } \\
\text { intervention. } \\
\text { Cost- } \\
\text { effectiveness: } \\
\text { Significant } \\
\text { differences at } 12 \\
\text { months, in } \\
\text { favour of } \\
\text { intervention }\end{array}$ \\
\hline $\begin{array}{l}\text { Rogers et } \\
\text { al (2009) }\end{array}$ & $\begin{array}{l}\text { Number of } \\
\text { sick days } \\
\text { in past } \\
\text { month: No } \\
\text { significant } \\
\text { differences }\end{array}$ & & & $\begin{array}{l}\text { Fatigue, Joint } \\
\text { pain, Physical } \\
\text { function: No } \\
\text { significant } \\
\text { differences } \\
\text { Physical } \\
\text { functioning } \\
\text { (item of FACT- } \\
\text { B): No } \\
\text { significant } \\
\text { differences } \\
\text { Joint } \\
\text { stiffness: } \\
\text { Significantly } \\
\text { greater } \\
\text { perceived } \\
\text { joint stiffness } \\
\text { in intervention } \\
\text { group } \\
\text { compared to } \\
\text { control. }\end{array}$ & $\begin{array}{l}\text { Emotional } \\
\text { functioning } \\
\text { (item of FACT- } \\
B \text { ): No } \\
\text { significant } \\
\text { differences }\end{array}$ & $\begin{array}{l}\text { QoL: } \\
\text { Significant } \\
\text { improvement } \\
\text { in social well- } \\
\text { being in } \\
\text { favour of } \\
\text { intervention } \\
\text { Not significant } \\
\text { for other QoL } \\
\text { outcomes }\end{array}$ & $\begin{array}{l}\text { Cognition, } \\
\text { Perceived health, } \\
\text { Endocrine } \\
\text { symptoms, Sleep } \\
\text { dysfunction: No } \\
\text { significant } \\
\text { differences }\end{array}$ \\
\hline
\end{tabular}

\section{Synthesis of Results}

Meta-analysis was possible for a limited number of studies for work-related outcomes: number of sick days taken, still on sick leave (yes/no), and working hours. However, because the majority of interventions, and reported outcome measures varied, narrative synthesis 
was also conducted. A summary of results of individual studies can be found in Table 3 .

\section{Effectiveness of interventions on work outcomes - meta-analysis}

Limited meta-analysis was possible on work outcomes including number of sick days taken (at six and 12-months) [17, 22], if someone remained on sick leave (at 12 months) [22, 24], and the number of working hours (at 12 months) $[24,28]$.

Number of sick days taken (six months and 12 months): Data for the number of sick days taken were available for two studies at six and 12 months [17, 22]. A random effects model was used due to heterogeneity between studies. At six months (Fig. 3), pooled analysis resulted in a non-significant overall effect of -0.08 (95\% Cl: $-0.48,0.38)$. Björneklett et al observed an effect close to zero of 0.03 (Hedge's $g$ ) between non-chemotherapy intervention and control groups (95\% Cl: -0.36, 0.42), and a small effect size of 0.26 (Hedge's g) between chemotherapy intervention and control groups (95\% Cl: $-0.13,0.65)$ [22]. Hubbard et al (2013) [17] observed a small effect size of -0.75 between intervention and control groups ( $\mathrm{Cl}$ : -1.70, 0.20). Figure 4 provides the results from meta-analysis of outcomes at twelve months. Björneklett et al observed an effect close to zero of 0.09 (Hedge's $\mathrm{g}$ ) between non-chemotherapy intervention and control groups (Cl: -0.33, 0.52) and a small effect size of 0.21 (Hedge's $g$ ) between chemotherapy intervention and control groups (95\% Cl: -0.20, 0.61) [22]. Hubbard et al observed a small effect size of -0.43 between intervention and control groups $(\mathrm{Cl}:-1.36,0.49)$ [17]. Pooled analysis indicated a nonsignificant overall very small effect of $0.10(\mathrm{Cl}:-0.28,0.39)$.

\section{Sick leave $(\mathrm{Y} / \mathrm{N})$ at 12 months - meta-analysis}

Sick leave data were available for two studies at 12 months [22, 24], however results were not statistically significant between intervention and control groups (Fig. 5). Björneklett et al observed an OR of 1.10 (95\% Cl: $0.57,2.12)$ for the association of any (vs no) sick leave between intervention vs control groups whereas Maunsell et al observed an OR of $1.13(95 \% \mathrm{Cl}$ : $0.48,2.68)$ for the associations of any sick leave between the intervention and control groups [22, 24]. Pooled analysis resulted in an overall OR (peto) of 1.11 (95\% Cl: $0.66,1.87$ ), which was close to 1.

Working hours at 12 months - meta-analysis

Working hours data were available for two studies at 12 months however, there was no significant evidence of a difference between the control and intervention groups (Fig. 6) [24, 28]. Maunsell et al observed an effect size close to zero of 0.05 (Hedge's $g$ ) between intervention and control groups ( $95 \% \mathrm{Cl}$ : $-0.20,0.30)$ whereas Mourgues et al observed a small-medium effect size of 0.4 (Hedge's $g$ ) between groups $(95 \% \mathrm{Cl}: 0.08,0.72)[24,28]$. Pooled analysis indicated an overall small effect of $0.19(95 \% \mathrm{Cl}$ : $-0.20,0.64)$. Heterogeneity measures indicated a $\mathrm{I}^{2}$ value of 28.27 and $\mathrm{H}^{2}$ value of 1.39. A random effects model for pooling analysis is shown in Fig. 6.

\section{Effectiveness of interventions on work outcomes - narrative synthesis}

Of the nine included studies, only one study reported statistically significant differences in favour of the intervention group for increased 'occupational activity' [28]. Findings indicated that the intervention group had significantly higher ability to perform work activities at 12months compared to the control group. The remaining eight studies did not report any statistically significant differences between groups, however increased numbers of the intervention group returning to work. or taking less sick leave compared to usual care, were reported in three studies; (i) the intervention group in Hubbard et al [17] study reported 53 fewer sick leave days compared to the control, (ii) Jong et al, [27] found $53 \%$ of the intervention group did RTW at six months compared to $23 \%$ of the control, and (iii) $76 \%$ of intervention group returned to work compared to $54 \%$ of the control in the study by Maguire et al, [23].

\section{Effectiveness of interventions on other health outcomes - narrative synthesis}

Other health outcomes were considered secondary outcomes in this review (Table 3).

Of the seven studies reporting physical outcomes, three reported statistically significant differences, which were not always positive. Rogers et al observed greater perceived joint stiffness in the intervention group compared to the control [21]. Four studies measured fatigue, two of which reported statistically significant differences in favour of the intervention group [25, 27]. While effect sizes in both studies were small, Bolam et al reported statistically significant differences between the RT-HIIT intervention and control groups in total cancer-related fatigue (CRF) and in Cognitive CRF [25]. While there were no statistically significant differences between groups in Multidimensional Fatigue Inventory (MFI) and Fatigue Quality List (FQL) scores in the Jong et al study, they did report a statistically significantly higher percentage of women in the intervention (51\%) experiencing fatigue reduction compared to the control (19\%) at 3months [27] 
Six studies reported psychological outcomes, only two of which demonstrated statistically significant results [23, 27]. Jong et al reported significant differences for the intervention group in depression at the three-month time point [27]. Maguire et al reported that participants in the control were statistically significantly more dissatisfied with scarring and prosthesis, than the intervention group [23].

Three out of four studies reported enhanced overall QoL, or components of QoL outcomes in favour of the intervention group [17, 21, 27]. While there were no statistically significant differences between groups in the total scores of the EORTC-QLQ-C30, Hubbard et al identified statistically significant differences between groups on the Breast Cancer Subscale at six-months, in favour of the intervention group [17]. Jong et al identified a statistically significant improvement in nausea and vomiting (an EORTC-QLQ-C30 item) for the intervention group at six-months [27]. Finally, Rogers et al observed statistically significant improvements in social wellbeing for the intervention group compared to control, with a large effect size of 0.76 [21].

\section{Cost-effectiveness - narrative synthesis}

Only two studies measured cost-effectiveness [22, 28]. Morgues et al, reported cost-effectiveness for the intervention at 12 months [28]. They examined direct (e.g., consultations, transport, thermal treatment) and indirect costs (e.g., out-of-pocket expenses associated with disease) in their cost effectiveness analysis (CEA). In contrast, Björneklett et al concluded that costs to society were not reduced with the intervention in its present form [22]. They reported total costs for the intervention group (cost of sick leave and consumption of health services) were higher at all time-points, reaching statistical significance between groups at 12 months. When adding the cost of the intervention $(€ 2,300)$ in addition to the costs of sick leave and healthcare utilisation, the costs for the intervention group were significantly higher at all time-points. While there were no significant differences between groups regarding visits to medical specialists, GPs or physiotherapists, women treated with chemotherapy in the intervention group had significantly more visits with 'other' healthcare professionals than the control group at 6 and 12 months.

\section{Discussion}

The objective of this review was to determine efficacy of rehabilitation interventions on work outcomes and identify core content and suitable measurement tools for interventions for women with breast cancer. The findings highlight variability across interventions for women with breast cancer, in intervention effectiveness, content, and delivery, currently available in published literature. Therefore, it is challenging to offer definitive recommendations on what constitutes an effective intervention to support work outcomes for women with breast cancer.

Only one study observed statistically significant differences in work outcomes between intervention, and control groups, observing greater resumption of work and participation in overall work activities at 12-months [28]. The success of this study could be partially explained by its multidisciplinary format providing exercise, psychological and dietary advice or to the sample size which may have been more adequately powered than other studies. A recent Cochrane review identified moderate quality evidence for multidisciplinary interventions in enhancing RTW rates across all cancer types [11]. Despite this, some aspects of the intervention (e.g., thermal water treatment) may be impractical if applied to informing a work-focused intervention, where thermal water treatment facilities are not widely available in healthcare services. In addition, no work-related content was included in the intervention. Lack of statistically significant impact on work outcomes across the other studies can perhaps be explained by the fact that the majority of interventions did not specifically focus on work in their interventions. Evidence suggests that interventions which are designed to target management of a specific concern, result in significant effects on that specified outcome [29]. While three studies in this current review included work components in their intervention, the content varied, and no statistically significant results were observed for work outcomes $[17,22,23]$. This could be because there was insufficient work-specific content in the interventions. Another explanation could be that the studies comprised of small sample sizes. For example, despite Hubbard et al including work-specific content in their intervention, only 18 women participated [17]. Future RCTs with larger samples may provide further insight into effectiveness using work-directed approaches. While work outcomes were measured across all studies by self-report, they varied from quantifying number of working days/hours to whether the participant had returned to work (yes/no response). Measuring RTW by binary yes/no could be problematic where the definition of RTW is blurred. As Lamore et al highlighted, RTW does not necessarily indicate that a previous lifestyle is completely restored, and there needs to be clarity as to the definition of RTW [30]. Researchers could consider if work outcomes imply RTW full-time or part-time, and perhaps perceived satisfaction with the outcome.

It is well documented that treatment and disease-related symptoms such as cancer-related fatigue, cognitive changes, and anxiety can impact on work ability and could be targeted as part of a RTW intervention $[9,10]$. Therefore, physical, psychological and QoL outcomes were also examined in this review. Outcomes differed widely across studies, with varying results making it challenging to offer definitive recommendations for the content and delivery of interventions to support return to work. Of the four studies measuring fatigue, significant

Page $18 / 26$ 
improvements were observed only in a physical intervention [25]. Interventions which deliver aerobic exercise have previously been cited in a Cochrane Review as beneficial in reducing cancer-related fatigue [31]. Another Cochrane Review reported limited evidence for psychosocial interventions in reducing fatigue unless specifically targeting fatigue [32]. An update of evidence is warranted however as the review was conducted more than a decade ago. In contrast, of the four studies measuring the impact of interventions on QoL, three which reported improvements, delivered both physical and psychosocial interventions. This underlines the importance of a multidisciplinary approach in RTW interventions in targeting a holistic range of treatment- and disease-related factors that impact on work. Interventions targeting QoL have varied considerably in participants, delivery and content making it difficult to arrive at a firm conclusion regarding effectiveness, although a Cochrane review tentatively concluded potential benefit of interventions which are educational and offer supportive attention [33]. Some specific outcomes of interest that are known to impact on work, were under-reported. For example, financial status, social support and cognitive dysfunction were less commonly reported outcomes, but could be considered, particularly as they can impact on RTW [8, 34]. In addition, considering upper limb function could be important for women with breast cancer, who are more likely to experience upper limb impairment compared to other cancer groups [8]. Lymphoedema, for example, is known to compound RTW challenges where there may be restrictions in mobility or heavy lifting, for example [35].

There also remain few studies reporting intervention cost-effectiveness. This gap is important to note as economic evaluation is a key consideration for decision-makers and is also outlined as a pillar for evaluation of complex interventions under the Medical Research Council framework for complex interventions [19]. Two of the nine studies reporting cost-effectiveness, observed contrasting results. One study observed higher costs for the intervention group who typically sought greater use of healthcare services than the control group [22]. This could be because women in the intervention received education on availability of healthcare professionals to assist with symptom management. Greater self-awareness of one's own health status could lead to a willingness to self-manage health and seek out appropriate health services. This could lead to reduced or self-managed co-morbidity in the future which could provide a cost-benefit for the intervention. In contrast, Mourgues et al observed enhanced work outcomes, and reported the intervention was cost-effective at 12 months [28]. It is not clear however if, like Björneklett et al, consultations with healthcare professionals other than medical professionals were also included in the analysis [22]. Mourgues et al did however use two facilitators as part of their intervention, whereas Björneklett et al use seven from a variety of disciplines $[22,28]$. This is likely to have impacted on the overall costs of each intervention. While multidisciplinary interventions have been identified as impacting on RTW rates in cancer care [11], researchers should take into consideration the overall cost impact if including a large range of disciplines. Future study designs could factor in healthcare utilisation into CEA both in the short- and long-term and avoid small sample sizes which are considered a limitation for calculating CEA.

On reviewing health behaviour change theory underlying study interventions, no clear conclusions on a preferred or most effective model can be drawn. Of the nine studies, only three reported using a theoretical framework, all of which varied. This gap has been previously echoed for other rehabilitation interventions for those with cancer $[8,30,36]$ and is noteworthy as incorporating insights from theory is recommended as a key consideration when developing complex interventions [19]. In this current review, none of the theories reported in the three studies were specific to work rehabilitation. For example, Social Cognitive Theory [37] which is often used in behaviour change interventions, was reported in one study [21]. This theory holds promise for understanding RTW motivations, expectations of efficacy, and predicting one's ability to achieve desired outcomes (i.e., work outcomes), but can be vague in operationalisation [38]. Similarly, while the Biopsychosocial model reported by Hubbard et al is holistic in nature considering biological, psychological and social factors, its generic nature can limit its direct application to work rehabilitation research and practice $[17,38,39]$. With this in mind, the evidence base beyond this current review can be explored for more specific models to occupational rehabilitation. A Cancer and Work Model was developed by Feuerstein et al, for all cancer cohorts, it includes factors that can be addressed by healthcare professionals, individuals living with or beyond cancer, and employers, and could be considered in intervention development [40].

This review provides an update on previous literature exploring return to work interventions for women with breast cancer where only one of four studies included in that review was controlled [15]. In contrast, all nine studies in this review were RCTs, potentially reducing selection bias. This is a promising indication that more rigorous methods are being employed in intervention evaluation. Most studies $(n=$ 6 ) in this current review were published since 2010 indicating growing research in recent years. Furthermore, a number of protocols for upcoming RCTs testing work interventions for women with breast cancer have been published [41-43], and it is likely that there will be an increased evidence-base to further explore feasibility and effectiveness in the future. There are however limitations in intervention development, where there is a lack of pilot and feasibility studies, which are advocated by several models for guiding intervention development $[19,44]$. Three of the nine studies in this current review were pilots, and the six remaining RCT studies did not report a pilot study prior to the full trial. While recruitment, adherence and attendance rates were referred to briefly in four studies [22, 24-26], feasibility was only explicitly reported in two $[17,21]$. Lack of piloting and feasibility research can lead to methodological challenges. For example, Jong et al [27] did not report any pilots or feasibility testing of their intervention and experienced recruitment issues during the RCT. Despite adding an additional recruitment site, recruitment remained challenging.

Page 19/26 
This review offers a collective insight into current evidence available on interventions for women with breast cancer that impact on work outcomes. A systematic search process was applied, limiting bias, and meta-analysis was possible for a number of outcomes which offers a statistical measure of the impact of intervention. Backwards and forwards chaining was completed on relevant texts to ensure complete inclusion of studies. Limitations were also identified. For practical reasons, a limit was applied to eligibility criteria for English-text only. However, this may have restricted other potential texts from being included in the final review. Meta-analysis was completed where possible, however, it is acknowledged that results need to be taken with caution as only two studies could be pooled for each analysis and the interventions examined might have been too heterogenous. There are numerous arguments for and against the meta-analysis of a small number of studies. Valentine and colleagues (2010) [45] argue, however, that given the need for a conclusion, two studies can be used for meta-analysis as all other synthesis techniques are less transparent. The study sizes were also small in several of the included studies, which may have limited the reliability and strength of evidence (power) to support the effectiveness of the interventions being evaluated.

\section{Recommendations for future practice and research}

In the absence of a sufficient evidence-base and the ability to make definitive recommendations, clinicians could consider multidisciplinary interventions to support women with breast cancer to return to work, as advocated by de Boer et al [11]. While rehabilitation interventions including work components did not observe statistically significant results, the value of work components cannot be ruled out, particularly where the only study to use a work-directed approach (e.g., work accommodations and modifications) was underpowered. Further research in developing and evaluating RTW interventions for women with breast cancer is warranted. Despite enhanced rigour in the study-designs over the past decade, there remains a paucity in piloting and testing feasibility of work-specific interventions. Future research could incorporate a model of intervention development into the study-design. Patient-reported work outcomes have typically been reported in studies. Objective measures (exploring work performance, for example) could also be considered in future designs. Furthermore, sufficient sample sizes to ensure an adequately powered study are necessary.

\section{Conclusion}

Interventions to support women with breast cancer to return to, or remain at, work remain scarce. Of the interventions that do exist, variability in content, and lack of evidence of the effectiveness on work outcomes, make it challenging to offer definitive recommendations for delivery of work-focused interventions. Despite this, studies of higher quality have emerged in the past decade with promising potential for an expanded evidence-base in the future. Future research in developing and evaluating work-focused interventions for women with breast cancer is warranted.

\section{Declarations}

Ethics approval and consent to participate: Not applicable

Consent for publication: Not applicable

Availability of data and materials: The datasets used and/or analysed during the current study available from the corresponding author on reasonable request.

Competing interests: The authors declare that they have no conflict of interest.

Funding: Naomi Algeo was supported with an Irish Research Council Government of Ireland Postgraduate Programme Scholarship during the time of conducting this research. Kathleen Bennett was supported by the Health Research Board (RL-15-1578).

Authors' contributions: N.A. screened all abstracts and titles for eligibility. All authors engaged in full-text review. N.A. and K.B. completed risk of bias assessment, and D.C. was available to resolve any disagreements. K.B. completed all statistical analysis. N.A. drafted the initial manuscript text and prepared figures 1-2, tables 1-3 and supplementary material. K.B. prepared figures 3-6. All authors reviewed the manuscript and approved the final version of the manuscript.

Acknowledgements: Naomi Algeo would like to thank the Irish Research Council who supported her with a Government of Ireland Postgraduate Programme Scholarship during the time of conducting this research. Prof. Kathleen Bennett would like to acknowledge support by the Health Research Board (RL-15-1578) during the time of this research. The authors would also like to thank Dr. Jan Hoving for providing additional information required for this review. 


\section{References}

1. GLOBOCAN. All cancers fact sheet. World Health Organization: 2018. Available at: https://gco.iarc.fr/today/data/factsheets/cancers/39-All-cancers-fact-sheet.pdf

2. American Cancer Society. Survival Rates for breast cancer. Atlanta: American Cancer Society. 2019. Available at: https://www.cancer.org/cancer/breast-cancer/understanding-a-breast-cancer-diagnosis/breast-cancer-survival-rates.html

3. Cancer Research UK. Breast cancer survival. London: Cancer Research UK. 2020. Available at: https://www.cancerresearchuk.org/about-cancer/breast-cancer/survival

4. National Cancer Registry Ireland. Cancer in Ireland 1994-2017 with estimates for 2017-2019: Annual report of the national cancer registry. National Cancer Registry Ireland. 2019. Available at: https://www.ncri.ie/sites/ncri/files/pubs/NCRI_Annual\%20Report2019_01102019.pdf

5. Parkinson M, Maheu C. Cancer and work. Canadian Oncology Nursing Journal. 2019;29:258-266. PMC6970012

6. Balak F, Roelen CA, Koopmans PC, Ten Berge EE, Groothoff JW. Return to work after early-stage breast cancer: a cohort study into the effects of treatment and cancer-related symptoms. J Occup Rehabil. 2008;18(3):267-272. doi: 10.1007/s10926-008-9146-z

7. Chaker L, Falla A, van der Lee SJ, Muka T, Imo D, Jaspers L, Colpani V, Mendis S, Chowdhury R, Bramer WM et al. The global impact of non-communicable diseases on macro-economic productivity: a systematic review. Eur J Epidemiol. 2015;30(5):357-395. doi: 10.1007/s10654-015-0026-5

8. Sun Y, Shigaki CL, Armer JM. Return to work among breast cancer survivors: a literature review. Support Care Cancer. 2017;25(3):709718. doi: $10.1007 / \mathrm{s} 00520-016-3446-1$

9. Todd BL, Feuerstein EL, Feuerstein M. When breast cancer survivors report cognitive problems at work. The International Journal of Psychiatry in Medicine. 2011;42(3):279-294. doi: 10.2190/PM.42.3.d

10. Carlsen K, Jensen AJ, Rugulies R, Christensen J, Bidstrup PE, Johansen C, Huitfeldt Madsen IE, Dalton So. Self-reported work ability in long-term breast cancer survivors. A

11. population-based questionnaire study in Denmark. Acta Oncol. 2013;52(2):423-429. doi:10.3109/0284186X.2012.744877

12. de Boer AGEM, Taskila TK, Tamminga SJ, Feuerstein M, Frings-Dresen MHW, Verbeek JH. Interventions to enhance return-to-work for cancer patients. Cochrane Database Syst Rev. [Internet]. 2015;(2). Available from:

https://www.cochranelibrary.com/cdsr/doi/10.1002/14651858.CD007569.pub3/full doi:

https://doi.org/10.1002/14651858.CD007569.pub3

13. Hegewald J, Wegewitz UE, Euler U, van Dijk JL, Adams J, Fista A, Heinrich P, Seidler A. Interventions to support return to work for people with coronary heart disease. Cochrane Database Sys Rev. 2019;3(Cd010748). doi: 10.1002/14651858.CD010748.pub2.

14. Fadyl JK, Anstiss D, Reed K, Khoronzhevych M, Levack WMM. Effectiveness of vocational interventions for gaining paid work for people living with mild to moderate mental health conditions: systematic review and meta-analysis. BMJ Open. 2020;10:e039699. doi: 10.1136/bmjopen-2020-039699

15. Nevala N, Pehkponen I, Teittinen A, Vesala HT, Pörtfors P, Anttila H. The effectiveness of rehabilitation interventions on the employment and functioning of people with intellectual disabilities: a systematic review. Journal of Occupational Rehabilitation. 2019;29(4):773-802. doi: 10.1007/s10926-019-09837-2

16. Hoving JL, Broekhuizen ML, Frings-Dresen MH. Return to work of breast cancer survivors: a systematic review of intervention studies. BMC Cancer. 2009;9(117):1-10. doi:10.1186/1471-2407-9-117

17. Moher D, Liberati A, Tetzlaff J, Altman DG, The PRISMA Group. Preferred Reporting Items for Systematic Reviews and Meta-Analyses: The PRISMA Statement. BMJ. 2009;6(7):e1000097. doi: 10.1136/bmj.b2535

18. Hubbard G, Gray NM, Ayansina D, Evans JMM, Kyle RG. Case management vocational rehabilitation for women with breast cancer after surgery: a feasibility study incorporating a pilot randomised controlled study. Trials. 2013;14(175). doi:10.1186/1745-6215-14175

19. Higgins JPT, Green S. Cochrane handbook for systematic reviews of interventions. 5th ed. The Cochrane Collaboration; 2011. Available at .

20. Craig P, Dieppe P, Macintyre S, Michie S, Nazareth I. Developing and evaluating complex interventions: the new Medical Research Council guidance. BMJ. 2008;337:a1655. doi: 10.1136/bmj.a1655

21. Von Hippel PT. The heterogeneity statistic $\mathrm{I}^{2}$ can be biased in small meta-analyses. BMC Medical Research Methodology. 2015;15(35). doi: 10.1186/s12874-015-0024-z 
22. Rogers LQ, Hopkins-Price P, Vicari S, Pamenter R, Courneya KS, Markwell S, Verhulst S, Hoelzer K, Naritoku C, Jones L, et al. A randomised trial to increase physical activity in breast cancer survivors. Medicine and Science in Sports and Exercise. 2009;41(4):935-946. doi: 10.1249/MSS.0b013e31818e0e1b

23. Björneklett HG, Rosenblad A, Lindemalm C, Ojutkangas $M-L$, Letocha H, Strang P, Bergkvist L. A randomized controlled trial of support group intervention after breast cancer treatment: Results on sick leave, health care utilization and health economy. Acta Oncologica. 2013;52(1):38-47. doi: 10.3109/0284186X.2012.734921

24. Maguire P, Brooke M, Tait A, Thomas C, Sellwood R. The effect of counselling on physical disability and social recovery after mastectomy. Clinical Oncology. 1983;9(4):319-324. Available at: https://pubmed.ncbi.nlm.nih.gov/6362943/

25. Maunsell E, Brisson J, Deschênes L, Frasure-Smith N. Randomized trial of a psychologic distress screening program after breast cancer: effects on quality of life. Journal of Clinical Oncology. 1996;14(10):2747-2755. doi: 10.1200/JC0.1996.14.10.2747

26. Bolam KA, Mijwel S, Rundqvist H, Wengström Y. Two-year follow-up of the OptiTrain randomised controlled exercise trial. Breast Cancer Research and Treatment. 2019;175(3):637-648. doi: https://doi.org/10.1007/s10549-019-05204-0

27. Ibrahim M, Muanza T, Smirnow N, Sateren W, Fournier B, Kavan P, Palumbo M, Dalfen R, Dalzell MA. Time course of upper limb function and return-to-work post-radiotherapy in young adults with breast cancer: a pilot randomized control trial on effects of targeted exercise program. J Cancer Surviv. 2017;11(6):791-799. doi: 10.1007/s11764-017-0617-0

28. Jong MC, Boers I, van der Velden Schouten AP, van der Meij S, Göker E, Timmer-Bonte ANJH, can Wietmarschen. A randomized study of yoga for fatigue and quality of life in women with breast cancer undergoing (neo) adjuvant chemo- therapy. J Alternat Complement Med. 2018;24(9):942-953. doi: 10.1089/acm.2018.0191

29. Mourgues C, Gerbaud L, Leger S, Auclair C, Peyrol F, Blanquet M, Kwiatkowski F, Leger-Enreille A, Bignon Y-J. Positive and costeffectiveness effect of spa therapy on the resumption of occupational and non-occupational activities in women in breast cancer remission: A french multicentre randomised controlled trial. European Journal of Oncology Nursing. 2014;18(2014):505-511. doi: 10.1016/j.ejon.2014.04.008

30. Howell D, Harth T, Brown J, Bennett C, Boyko S. Self-management education interventions for patients with cancer: a systematic review. Supportive Care in Cancer. 2017;25(4):1323-1355. doi: 10.1007/s00520-016-3500-z

31. Lamore K, Dubois T, Rothe U, Leonardi M, Girard I, Manuwald U et al. Return to work interventions for cancer survivors: a systematic review and a methodological critique. Int J Environ Res Public Health. 2019;16(8):1343. doi: 10.3390/ijerph16081343

32. Cramp F. \& Byron-Daniel J. Exercise for the management of cancer-related fatigue in adults. Cochrane Database of Systematic Reviews. 2012. Available at: https://doi.org/10.1002/14651858.CD006145.pub3

33. Goedendorp MM, Gielissen MFM, Verhagen CAHHVM, Bleijenberg G. Psychosocial interventions for reducing fatigue during cancer treatment in adults. Cochrane Database of Systematic Reviews. 2009. Available at:

https://doi.org/10.1002/14651858.CD006953.pub2

34. Galway K, Black A, Cantwell M, Cardwell CR, Mills M \& Donnelly M. Psychosocial interventions to improve quality of life and emotional wellbeing for recently diagnosed cancer patients. Cochrane Database of Systematic Reviews. 2012. Available at: https://doi.org/10.1002/14651858.CD007064.pub2

35. Islam T, Dahlui M, Abd Majid H, Nahar AM, Taib N, Su TT, et al. Factors associated with return to work of breast cancer survivors: a systematic review. BMC Public Health. 2014;14(Suppl 3):S8. doi: 10.1186/1471-2458-14-S3-S8

36. Boyages J, Kalfa S, Xu Y, Koelmeyer L, Mackie H, Viveros H, Taksa L, Gollan P. Worse and worse off: the impact of lymphedema on work and career after breast cancer. SpringerPlus. 2016;5(1):1-8. doi:10.1186/s40064-016-2300-8

37. Désiron HAM, Donceel P, de Rijk A, et al. A conceptual-practice model for occupational therapy to facilitate return to work in breast cancer patients. J Occup Rehabil. 2013;23(4):516-526. doi: 10.1007/s10926-013-9427-z

38. Bandura A. Social foundations of thought and action: a social cognitive theory. New Jersey: Prentice-Hall; 1986.

39. Schultz IZ, Stowell AW, Feuerstein M, Gatchel RJ. Models of return to work for musculoskeletal disorders. J Occup Rehabil. 2007;17(2):327-352. doi: 10.1007/s10926-007-9071-6

40. Engel GL. The need for a new medical model: a challenge for biomedicine. Science. 1977;196(4286):129-136. doi: $10.1126 /$ science. 847460

41. Feuerstein M, Todd BL, Moskowitz MC, Bruns GL, Stoler MR, Nassif T, Yu X. Work in cancer survivors: a model for practice and research. J Cancer Surviv. 2010;4(4):415-437. doi: 10.1007/s11764-010-0154-6

42. Stevens CL, Hegel MT, Bakitas MA, et al. Study protocol for a multisite randomised controlled trial of a rehabilitation intervention to reduce participation restrictions among female breast cancer survivors. BMJ Open. 2020;10:e036864. doi: 10.1136/bmjopen-2020-

Page 22/26 
036864

43. Sheppard DM, Frost D, Jefford M, O'Connor M, Halkett G. 'Beyond Cancer': a study protocol of a multimodal occupational rehabilitation programme to support breast cancer survivors to return to work. BMJ Open. 2019;9:e032505. doi: 10.1136/bmjopen2019-032505

44. de Groef A., Devoogdt N, Van der Gucht E. et al. EduCan trial: study protocol for a randomised controlled trial on the effectiveness of pain neuroscience education after breast cancer surgery on pain, physical, emotional and work-related functioning. BMJ Open. 2019;9:e025742. doi:10.1136/ bmjopen-2018-025742

45. Czajkowski SM, Powell LH, Adler N, Naar-King S, Reynolds KD, et al. From ideas to efficacy: the ORBIT model for developing behavioral treatments for chronic diseases. Health Psychol. 2015;34(10):971-82. doi: 10.1037/hea0000161

46. Valentine JC, Pigott TD, Rothstein HR. How many studies do you need? A primer on statistical power for meta-analysis. Journal of Educational and Behavioural Statistics. 2010;35(2):215-247. doi: 10.3102/1076998609346961

\section{Figures}

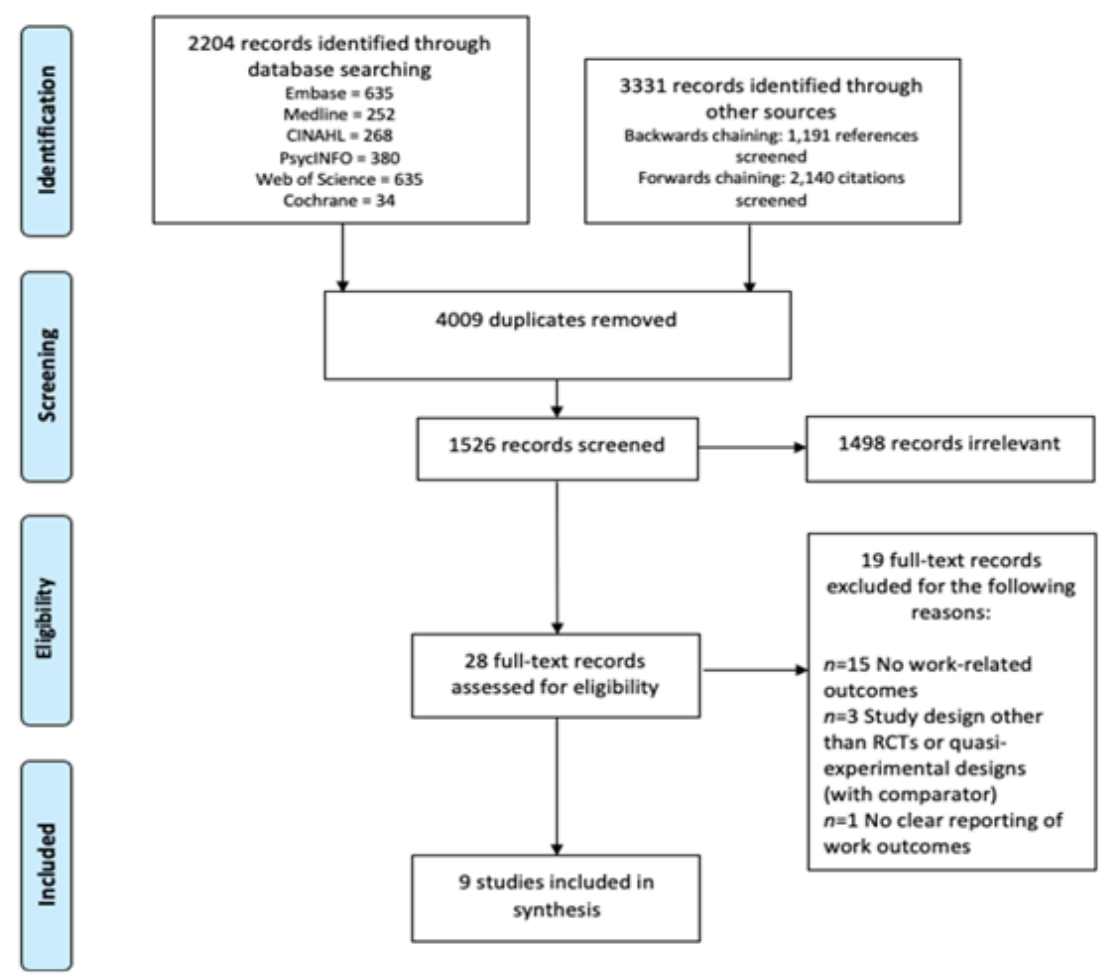

Figure 1

PRISMA Flow Diagram 


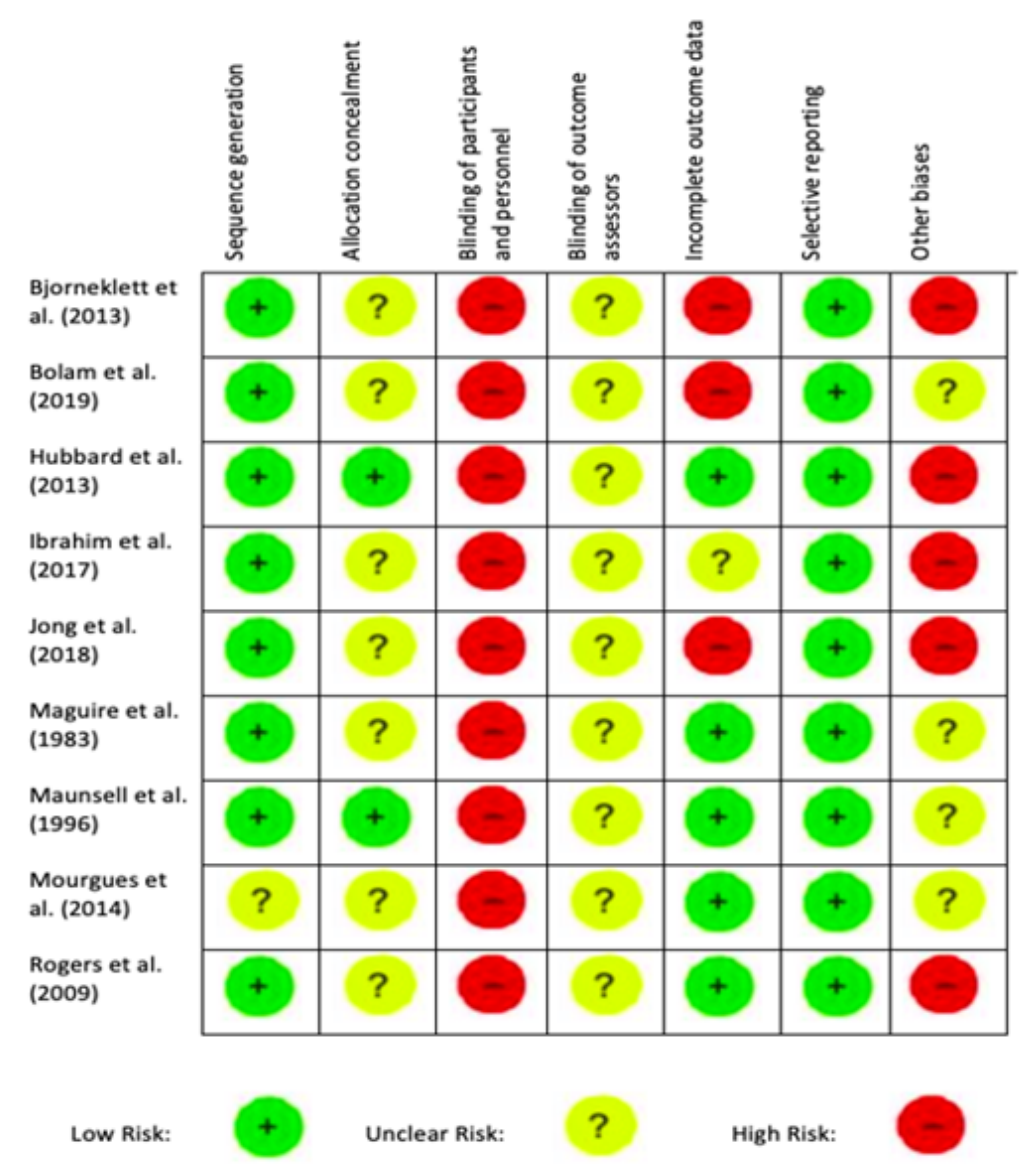

Figure 2

Risk of Bias Assessment

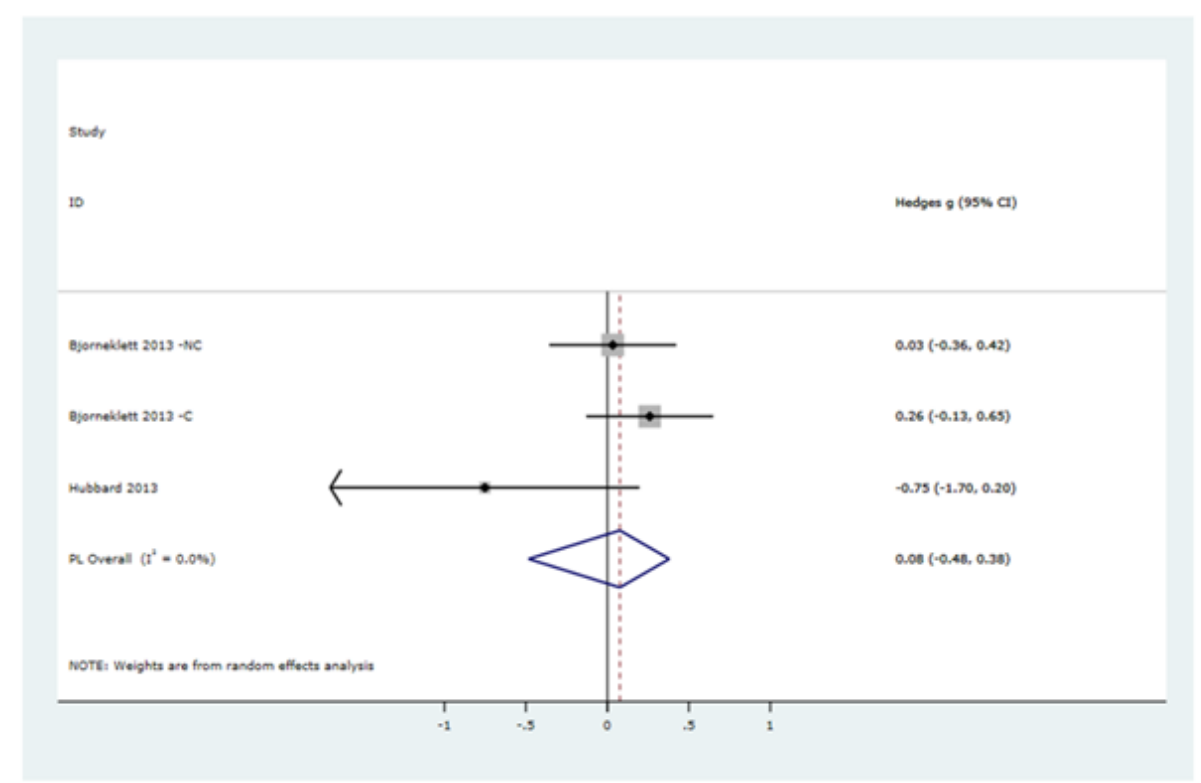

\section{Figure 3}

Meta-analysis of number of sick days taken at six months. NC: Non-Chemotherapy Group; C: Chemotherapy Group 
study

10

Hedges o $(95 \% \mathrm{C})$

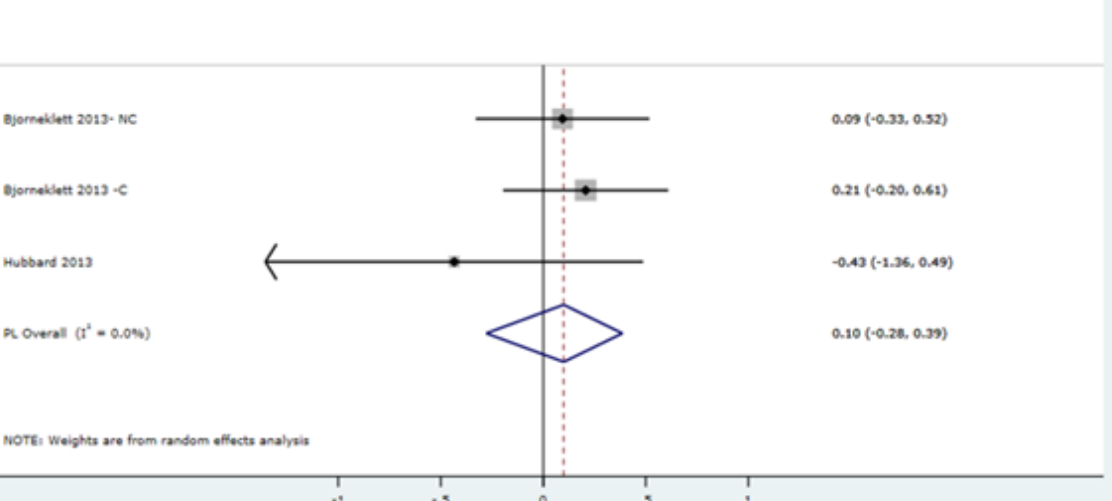

\section{Figure 4}

Meta-analysis of number of sick days taken at twelve months. NC: Non-Chemotherapy Group; C: Chemotherapy Group

Study

ID

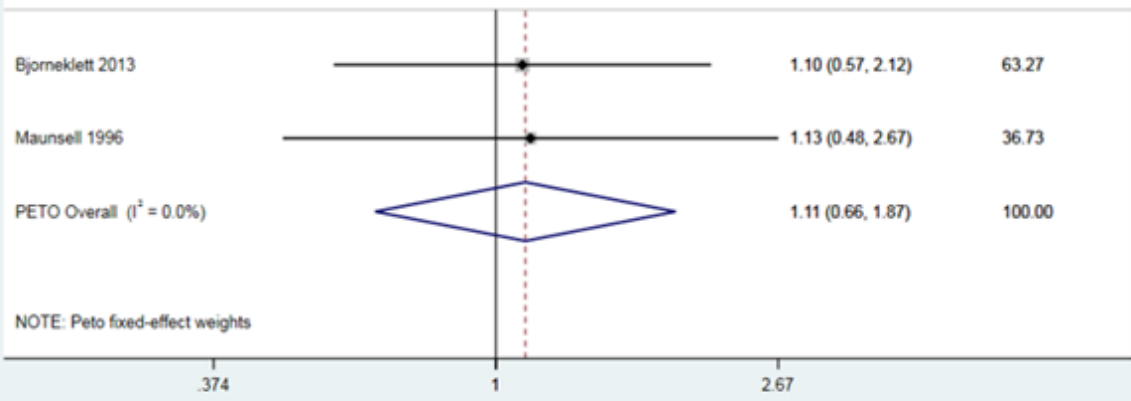

\section{Figure 5}

Meta-analysis of sick leave (Yes/No) at twelve months 


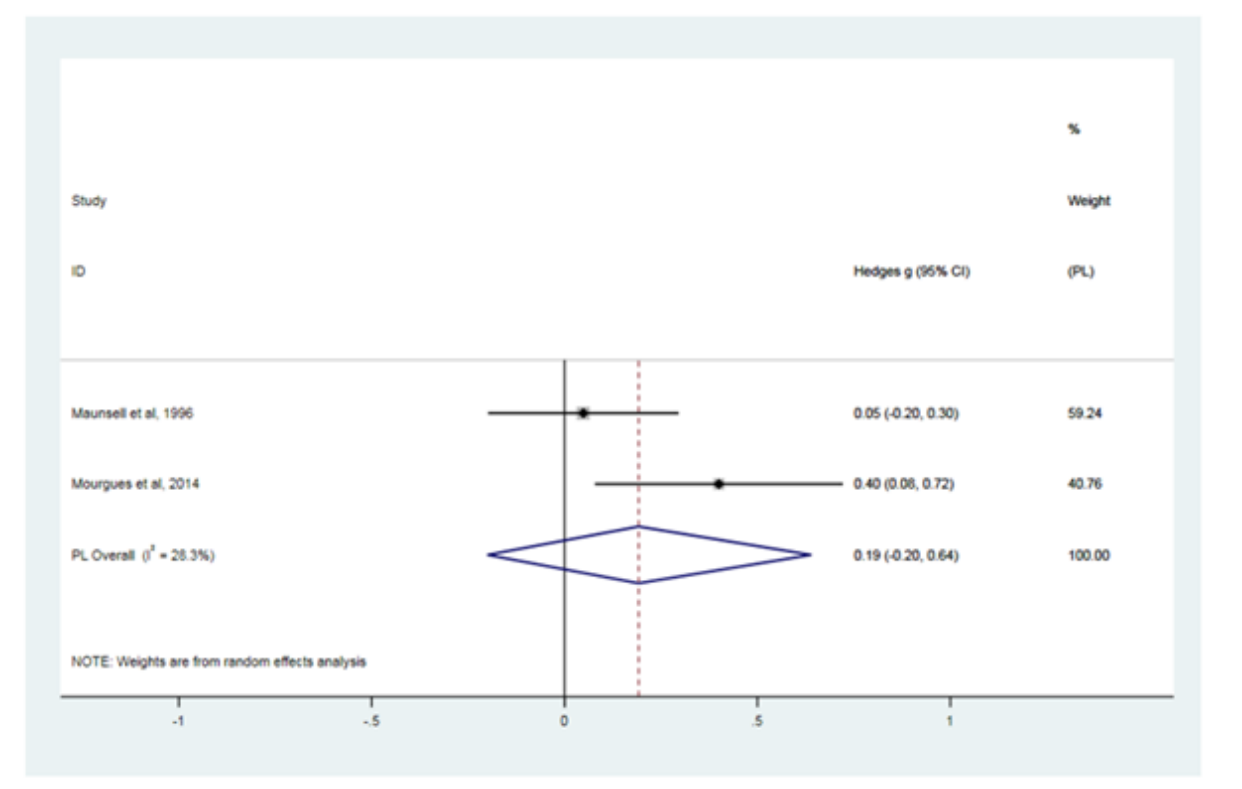

\section{Figure 6}

Meta-analysis of working hours at twelve months

\section{Supplementary Files}

This is a list of supplementary files associated with this preprint. Click to download.

- SupplementaryMaterial.docx 